\title{
Loop corrections to the power spectrum for massive neutrino cosmologies with full time- and scale-dependence
}

\author{
Mathias Garny, Petter Taule \\ Physik Department T31, \\ James-Franck-Str. 1, Technische Universität München, \\ D-85748 Garching, Germany \\ E-mail: mathias.garny@tum.de, petter.taule@tum.de
}

\begin{abstract}
Loop corrections to the power spectrum are frequently computed using approximate non-linear kernels adopted from an Einstein de-Sitter (EdS) cosmology. We present an algorithm that allows us to take the full time- and scale-dependence of the underlying fluid dynamics into account, and apply it to assess the impact of neutrino free-streaming on the 1- and 2-loop matter power spectrum. Neutrino perturbations are described by a hybrid Boltzmann/two-fluid model, that we show to agree with the CLASS Boltzmann solution at the linear level when using an appropriate effective neutrino sound velocity. We then use this scheme at 1- and 2-loop to perform a precision comparison of the matter power spectrum with simplified treatments of massive neutrinos. For a commonly adopted scheme using cold dark matter+baryon perturbations and EdS kernels to compute non-linear corrections, we find deviations above $1 \%$ for $k>0.15 h / \mathrm{Mpc}$ at $z=0$, and sub-percent agreement on weakly non-linear scales at $z=0.5$. We also demonstrate the impact of the EdS approximation on the 2-loop power spectrum for massless neutrinos.
\end{abstract}




\section{Contents}

1 Introduction 1

2 Evaluation of loop corrections with exact time- and scale-dependence $\quad 3$

2.1 Standard Perturbation Theory 3

2.2 Extension beyond SPT and numerical evaluation 4

$\begin{array}{lll}3 & \text { Validity of EdS approximation } & 7\end{array}$

4 Two-fluid model: CDM + baryons and massive neutrinos 9

$\begin{array}{lll}4.1 & \text { Sound velocity and anisotropic stress } & 11\end{array}$

4.2 Comparison with Boltzmann solver 13

5 Non-linear power spectrum with massive neutrinos $\quad 16$

$\begin{array}{ll}5.1 \text { Approximation schemes } & 17\end{array}$

$\begin{array}{lll}5.2 \text { Comparison } & 19\end{array}$

6 Conclusions 23

A Validation with analytic 1-loop kernels 24

B Numerical checks of convergence 26

\section{Introduction}

Mapping out the large-scale structure (LSS) of the Universe is one of the key advances that is driving precision cosmology, and current [1-3] and near future [4-7] surveys will allow us to potentially detect even small deviations from the $\Lambda \mathrm{CDM}$ model at the (sub-)percent level. Apart from testing possible explanations of the apparent tension in the value of the Hubble constant $H_{0}$ within extended cosmological models, precise LSS observations enable us to scrutinize the properties of dark energy or probe tiny deviations from the cold dark matter hypothesis on large scales $[4,8,9]$. Indeed, even within the standard cosmological model, the non-baryonic matter is expected to be composed of a mixture of cold and hot components, the latter being provided by the cosmic neutrino background. Identifying its impact on structure formation offers the prospect to infer the absolute neutrino mass scale within the $\Lambda$ CDM framework $[4,10-15]$.

A major technical challenge when exploiting LSS data is to provide precise and robust theoretical predictions for a large set of cosmological parameters, as required in a statistical analysis. Given the extended coverage of weakly non-linear scales in future surveys, major efforts have been devoted to obtain a suitable perturbative description [16]. Useful ingredients that have been explored in this context include the perturbative bias expansion [17], models of redshift-space distortions (RSD) [18], analyses of stress-tensor contributions [19], effective field theory methods [20], as well as IR resummation capturing the non-linear broadening of the baryon acoustic oscillation (BAO) feature in the two-point correlation function [21-24]. A viable analysis strategy consists in capturing bias, RSD as well as the impact of small (UV) scales by a number of free parameters, that can be marginalized over. Approaches 
along these lines have, in various implementations that differ in the underlying model but yield consistent results, been applied to analyze the full shape of BOSS galaxy clustering data $[25-28]$.

To achieve a theoretical uncertainty at or below the percent level, every element of the perturbative description needs to be scrutinized. In this work we focus on the impact of timeand scale-dependence of the growth of structures on the computation of non-linear corrections to the power spectrum. While the framework that we present here is applicable to a large variety of extended cosmological models, we discuss in particular the case of massive neutrinos. Within the framework of standard perturbation theory (SPT), the density contrast is expanded perturbatively around the linear solution, yielding wavenumber-dependent kernels $F_{n}\left(\mathbf{k}_{1}, \ldots, \mathbf{k}_{n}\right)$ at the $n$-th order. In turn, these kernels furnish the perturbative expansion of the power spectrum. A major simplification occurs for an Einstein de-Sitter (EdS) Universe that is dominated by matter. In this case the time-dependence of the kernels factorizes, and they can be computed using well-known algebraic recursion relations. This simplification is in general not possible in any realistic cosmological model, including $\Lambda$ CDM. Nevertheless, EdS-SPT kernels are widely used also for $\Lambda$ CDM, after correcting for the main impact of dark energy by using a suitably rescaled time variable [16].

When including massive neutrinos, the linear growth rate becomes scale-dependent due to neutrino free streaming [29]. An accurate description at the linear level requires to solve Boltzmann equations for the neutrino perturbations, as is done routinely in codes such as CLASS [30] or CAMB [31]. However, this quickly becomes impractical beyond linear perturbation theory [32]. Fortunately, for values of the neutrino mass targeted by future surveys, their distribution becomes non-relativistic long before non-linear corrections become relevant. This enables a hybrid description based on the full Boltzmann evolution at high redshifts, and an effective two-fluid description at low redshift, that is amenable to perturbation theory [33]. In this work we present an improved version of the two-fluid description that reproduces the linear evolution predicted by the Boltzmann equation at the permille level for cold dark matter, and with better than percent accuracy for neutrino perturbations. Given that the latter constitute only a small fraction of the total density contrast, this translates into a negligible error for the matter power spectrum. Free-streaming is captured by an effective, scale-dependent sound velocity within the fluid description. Based on this two-fluid model, we obtain results for the 1- and 2-loop power spectrum, taking the exact time- and scale-dependence of the underlying fluid dynamics into account. We compare these results to various simplified approximation schemes for massive neutrinos that are commonly used in this context, and assess in how far they capture the impact of free-streaming. Our work should be considered as a starting point, aiming at an inclusion of the effects of redshift space distortions, stress-tensor/effective field theory corrections as well as relativistic effects [34] in the future. In addition, the framework for computing non-linear corrections employed in this work is rather versatile and can be applied to extended cosmological models with scaledependent growth in the future. Aiming at an accurate and efficient description within the weakly non-linear regime, the perturbative approach is complementary to $N$-body simulations [35-37] and (halo-)models [38] investigating the effect of massive neutrinos on smaller scales.

In Sec.2, we introduce the general setup for computing loop corrections to the power spectrum taking exact time- and scale-dependence into account, keeping the discussion as general as possible. A first application to $\Lambda \mathrm{CDM}$ is discussed in Sec. 3, where we assess the error of the EdS-SPT approximation at 2-loop. We turn to massive neutrinos in Sec. 4, where 
we introduce the hybrid Boltzmann/two-fluid description and quantify its accuracy at the linear level. In Sec.5, we present results for the 1- and 2-loop power spectrum for massive neutrinos and compare them to various approximate schemes, before concluding in Sec. 6. In the appendices we discuss the validation and convergence checks of our numerical algorithm.

\section{Evaluation of loop corrections with exact time- and scale-dependence}

In this section we discuss the strategy for computing loop corrections to the power spectrum with exact time- and scale-dependence that allows us to go up to 2-loop. After briefly reviewing the standard formalism and setting up our notations, we introduce a generic extension of standard perturbation theory that can capture a large variety of cosmological models beyond $\Lambda \mathrm{CDM}$, as well as effective descriptions of structure formation. We then outline the algorithm for computing non-linear corrections to the power spectrum within this framework, leaving some details relevant for the application to massive neutrinos to Sec. 5 .

\subsection{Standard Perturbation Theory}

We start by briefly reviewing the formalism for standard perturbation theory, and introducing some basic notations. The equations of motion for the density contrast $\delta$ and velocity divergence $\theta \equiv \partial_{i} \mathbf{v}^{i}$ (neglecting vorticity) in Fourier space are

$$
\begin{aligned}
\partial_{\tau} \delta(\mathbf{k}, \tau)+\theta(\mathbf{k}, \tau) & =-\int_{\mathbf{k}_{1}, \mathbf{k}_{2}} \delta_{D}\left(\mathbf{k}-\mathbf{k}_{12}\right) \alpha\left(\mathbf{k}_{1}, \mathbf{k}_{2}\right) \theta\left(\mathbf{k}_{1}, \tau\right) \delta\left(\mathbf{k}_{2}, \tau\right), \\
\partial_{\tau} \theta(\mathbf{k}, \tau)+\mathcal{H} \theta(\mathbf{k}, \tau)+\frac{3}{2} \mathcal{H}^{2} \Omega_{m} \delta(\mathbf{k}, \tau) & =-\int_{\mathbf{k}_{1}, \mathbf{k}_{2}} \delta_{D}\left(\mathbf{k}-\mathbf{k}_{12}\right) \beta\left(\mathbf{k}_{1}, \mathbf{k}_{2}\right) \theta\left(\mathbf{k}_{1}, \tau\right) \theta\left(\mathbf{k}_{2}, \tau\right),
\end{aligned}
$$

where $\tau$ is conformal time, $\mathcal{H}=\mathrm{d} \ln a / \mathrm{d} \tau, \Omega_{m}$ the time-dependent matter density parameter, $\delta_{D}$ denotes the Dirac delta function and we use the shorthand notations $\mathbf{k}_{12}=\mathbf{k}_{1}+\mathbf{k}_{2}$ and $\int_{\mathbf{k}}=\int \mathrm{d}^{3} \mathbf{k}$. The mode coupling functions are

$$
\alpha\left(\mathbf{k}_{1}, \mathbf{k}_{2}\right)=1+\frac{\mathbf{k}_{1} \cdot \mathbf{k}_{2}}{k_{1}^{2}}, \quad \beta\left(\mathbf{k}_{1}, \mathbf{k}_{2}\right)=\frac{\left(\mathbf{k}_{1}+\mathbf{k}_{2}\right)^{2}\left(\mathbf{k}_{1} \cdot \mathbf{k}_{2}\right)}{2 k_{1}^{2} k_{2}^{2}} .
$$

Introducing the tuple $\psi_{a}=(\delta,-\theta / \mathcal{H} f)$, the above equations may be written in the compact form

$$
\partial_{\eta} \psi_{a}(\mathbf{k}, \eta)+\Omega_{a b}(\eta) \psi_{b}(\mathbf{k}, \eta)=\int_{\mathbf{k}_{1}, \mathbf{k}_{2}} \delta_{D}\left(\mathbf{k}-\mathbf{k}_{12}\right) \gamma_{a b c}\left(\mathbf{k}, \mathbf{k}_{1}, \mathbf{k}_{2}\right) \psi_{b}\left(\mathbf{k}_{1}, \eta\right) \psi_{c}\left(\mathbf{k}_{2}, \eta\right),
$$

where $\eta=\ln D$ and $f=\mathrm{d} \ln D / \mathrm{d} \ln a, D$ being the linear growth factor. The matrix $\Omega_{a b}$ is given by

$$
\Omega_{a b}(\eta)=\left(\begin{array}{cc}
0 & -1 \\
-\frac{3}{2} \frac{\Omega_{m}}{f^{2}} & \frac{3}{2} \frac{\Omega_{m}}{f^{2}}-1
\end{array}\right),
$$

and the only non-zero elements of $\gamma_{a b c}$ are

$$
\gamma_{121}\left(\mathbf{k}, \mathbf{k}_{1}, \mathbf{k}_{2}\right)=\alpha\left(\mathbf{k}_{1}, \mathbf{k}_{2}\right), \quad \gamma_{222}\left(\mathbf{k}, \mathbf{k}_{1}, \mathbf{k}_{2}\right)=\beta\left(\mathbf{k}_{1}, \mathbf{k}_{2}\right) .
$$

In an EdS Universe, $\Omega_{m}=1$ and $f=1$, which makes $\Omega_{a b}$ time-independent. Even though $\Omega_{m}$ and $f$ differ significantly from unity entering vacuum energy domination for a 
$\Lambda \mathrm{CDM}$ or $w \mathrm{CDM}$ cosmology, the ratio $\Omega_{m} / f^{2}$ remains close to 1 . In addition, at the linear level, the deviation of $\Omega_{m} / f^{2}$ from unity does not affect the growing mode (with eigenvector proportional to $(1,1)$ ). This is usually taken as an argument to replace the matrix $\Omega_{a b}$ by

$$
\Omega_{a b}^{\mathrm{EdS}}(\eta)=\left(\begin{array}{cc}
0 & -1 \\
-\frac{3}{2} & \frac{1}{2}
\end{array}\right)
$$

even when considering $\Lambda \mathrm{CDM}$ cosmology, leading to the conventional form of SPT, that we denote by EdS-SPT. The virtue of this approximation is that, when solving (2.3) perturbatively in the linear density contrast $\delta^{(1)}(\mathbf{k}, \eta)=\mathrm{e}^{\eta} \delta_{0}(\mathbf{k})$, the time-dependence factorizes,

$$
\delta(\mathbf{k}, \eta)=\sum_{n=1}^{\infty} \int_{\mathbf{q}_{1}, \ldots, \mathbf{q}_{n}} \delta_{D}\left(\mathbf{k}-\mathbf{q}_{1 \cdots n}\right) \mathrm{e}^{n \eta} F_{n}\left(\mathbf{q}_{1}, \ldots, \mathbf{q}_{n}\right) \delta_{0}\left(\mathbf{q}_{1}\right) \cdots \delta_{0}\left(\mathbf{q}_{n}\right),
$$

where $\mathbf{q}_{1 \cdots n}=\sum_{i} \mathbf{q}_{i}$. A similar expansion holds for $\psi_{2}=-\theta / \mathcal{H} f$ with kernels $G_{n}$. The EdS-SPT kernels can be obtained from well-known algebraic recursion relations [16], and furnish the perturbative expansion of the power spectrum

$$
\left\langle\delta(\mathbf{k}, \eta) \delta\left(\mathbf{k}^{\prime}, \eta\right)\right\rangle=\delta_{D}\left(\mathbf{k}+\mathbf{k}^{\prime}\right) P(k, \eta) .
$$

The EdS-SPT kernels are frequently used also for models beyond $\Lambda$ CDM, and in particular for massive neutrinos. In this work we scrutinize this approximation using the framework described below.

\subsection{Extension beyond SPT and numerical evaluation}

Going beyond EdS-SPT generically leads to a breakdown of the factorization of time- and scale-dependence when expanding the density contrast perturbatively. This poses a significant technical increase in complexity, and has therefore been little explored so far beyond 1-loop. In this section we present the setup that we employ to tackle this challenge. The algorithm is an extension of the one employed in [39-41].

We start by introducing a generic extension of SPT that potentially encompasses many models beyond $\Lambda \mathrm{CDM}$ as well as effective descriptions of structure formation. Below we will apply this setup to massive neutrino cosmologies, and also assess the accuracy of the EdS approximation for $\Omega_{a b}$ within $\Lambda$ CDM. We assume that cosmic perturbations can be described by a set of fields $\psi_{a}(\mathbf{k}, \eta)$, allowing for multiple species. For an $N$-fluid model the vector $\psi_{a}$ contains the density contrast $\delta_{i}$ and the (suitably normalized) divergence of the peculiar velocity field $\theta_{i}$ for each fluid component $i$, and the index $a$ runs from 1 to $2 N$. The full set of auto- and cross power spectra of these perturbations is given by

$$
\left\langle\psi_{a}(\mathbf{k}, \eta) \psi_{b}\left(\mathbf{k}^{\prime}, \eta\right)\right\rangle=\delta_{D}\left(\mathbf{k}+\mathbf{k}^{\prime}\right) P_{a b}(k, \eta) .
$$

We further assume that the non-linear equation of motion of $\psi_{a}$ can be brought into a form analogous to Eq. (2.3),

$$
\partial_{\eta} \psi_{a}(\mathbf{k}, \eta)+\Omega_{a b}(k, \eta) \psi_{b}(\mathbf{k}, \eta)=\int_{\mathbf{k}_{1}, \mathbf{k}_{2}} \delta_{D}\left(\mathbf{k}-\mathbf{k}_{12}\right) \gamma_{a b c}\left(\mathbf{k}, \mathbf{k}_{1}, \mathbf{k}_{2}\right) \psi_{b}\left(\mathbf{k}_{1}, \eta\right) \psi_{c}\left(\mathbf{k}_{2}, \eta\right) .
$$

The left-hand side comprises the linear equation of motion, being captured by a $2 N \times 2 N$ matrix $\Omega_{a b}(k, \eta)$. We allow for both a time- and wavenumber-dependence, with $k \equiv|\mathbf{k}|$. The 
dependence on wavenumber occurs for example when taking an effective viscosity and sound velocity into account for dark matter [41], and is also important for massive neutrinos [33]. In addition, $\gamma_{a b c}\left(\mathbf{k}, \mathbf{k}_{1}, \mathbf{k}_{2}\right)$ denote the non-linear vertices, that capture quadratic non-linearities in the equations of motion in real space. For $\Lambda$ CDM they are given by Eq. (2.5), but may also include additional non-linear terms in general.

The equation of motion needs to be complemented by suitable initial conditions. We assume that the initial conditions for all perturbation variables are correlated, as is the case for the familiar adiabatic initial conditions. Consequently, they can be brought into the form

$$
\psi_{a}\left(\mathbf{k}, \eta_{\text {ini }}\right)=u_{a}\left(k, \eta_{\text {ini }}\right) \delta_{0}(\mathbf{k}),
$$

where $\eta_{\text {ini }}$ should be chosen sufficiently late after recombination, but long before the onset of non-linear evolution on scales relevant for large-scale structure formation. Neglecting primordial non-Gaussianity, $\delta_{0}(\mathbf{k})$ can be taken as a Gaussian random field, of which we ultimately only need to know the (linear) power spectrum $\left\langle\delta_{0}(\mathbf{k}) \delta_{0}\left(\mathbf{k}^{\prime}\right)\right\rangle=\delta_{D}\left(\mathbf{k}+\mathbf{k}^{\prime}\right) P_{0}(k)$ as an input for the computation of non-linear corrections. The vector $u_{a}\left(k, \eta_{\text {ini }}\right)$ determines the relative normalization of the initial values for the various perturbation variables. For a $\Lambda \mathrm{CDM}$ cosmology featuring only a single fluid component, it can be chosen to agree with the growing mode eigenvector $u_{a}\left(k, \eta_{\text {ini }}\right)=(1,1)$ with $\eta_{\text {ini }}$ deep inside the matter dominated era. We refer to the following sections for some details on how the initial conditions are implemented for the respective applications.

The perturbative expansion of the solution to Eq. (2.10) can be written in the form

$$
\psi_{a}(\mathbf{k}, \eta)=\sum_{n=1}^{\infty} \int_{\mathbf{q}_{1}, \ldots, \mathbf{q}_{n}} \delta_{D}\left(\mathbf{k}-\mathbf{q}_{1 \cdots n}\right) F_{a}^{(n)}\left(\mathbf{q}_{1}, \ldots, \mathbf{q}_{n} ; \eta\right) \delta_{0}\left(\mathbf{q}_{1}\right) \cdots \delta_{0}\left(\mathbf{q}_{n}\right)
$$

Importantly, compared to the EdS-SPT case given in Eq. (2.7), the time-dependence does in general not factorize. To obtain an evolution equation for the kernels, we insert Eq. (2.12) into the equation of motion (2.10) and collect terms of equal order in $\delta_{0}$. This yields an equation of motion for the kernels

$$
\begin{aligned}
& \partial_{\eta} F_{a}^{(n)}\left(\mathbf{q}_{1}, \ldots, \mathbf{q}_{n} ; \eta\right)+\Omega_{a b}(k, \eta) F_{b}^{(n)}\left(\mathbf{q}_{1}, \ldots, \mathbf{q}_{n} ; \eta\right) \\
& =\sum_{m=1}^{n-1}\left[\gamma_{a b c}\left(\mathbf{k}, \mathbf{q}_{1 \cdots m}, \mathbf{q}_{m+1 \cdots n}\right) F_{b}^{(m)}\left(\mathbf{q}_{1}, \ldots, \mathbf{q}_{m} ; \eta\right) F_{c}^{(n-m)}\left(\mathbf{q}_{m+1}, \ldots, \mathbf{q}_{n} ; \eta\right)\right]_{\mathrm{sym} .}
\end{aligned}
$$

Here the right hand side is understood to be symmetrized with respect to $N_{m}=\frac{n !}{m !(n-m) !}$ permutations exchanging momenta in the $\left\{\mathbf{q}_{1}, \ldots, \mathbf{q}_{m}\right\}$ set with momenta in the $\left\{\mathbf{q}_{m+1}, \ldots, \mathbf{q}_{n}\right\}$ set (meaning the sum of all permuted expressions divided by $N_{m}$ ). Furthermore, $\mathbf{k}=\sum_{i} \mathbf{q}_{i}$.

For any given set of wavevectors, Eq. (2.13) is a set of coupled ordinary differential equation for the $2 N$ kernels $F_{a}^{(n)}\left(\mathbf{q}_{1}, \ldots, \mathbf{q}_{n} ; \eta\right)$ with $a=1, \ldots, 2 N$. One can easily check that for $N=1$, when replacing $\Omega_{a b}(k, \eta)$ by the constant EdS matrix given in Eq. (2.6), along with the vertices from Eq. (2.5), one recovers the usual EdS-SPT recursion relations for the kernels in the limit $\eta_{\text {ini }} \rightarrow-\infty$, with a factorized time-dependence $F_{1}^{(n)} \rightarrow \mathrm{e}^{n \eta} F_{n}$ and $F_{2}^{(n)} \rightarrow \mathrm{e}^{n \eta} G_{n}$.

Inserting the perturbative expansion Eq. (2.12) into Eq. (2.9) and using the Wick theorem yields the "loop" expansion of the power spectrum

$$
P_{a b}(k, \eta)=P_{a b}^{\operatorname{lin}}(k, \eta)+P_{a b}^{1 \text {-loop }}(k, \eta)+P_{a b}^{2 \text {-loop }}(k, \eta)+\ldots,
$$


with

$$
\begin{aligned}
P_{a b}^{\operatorname{lin}}(k, \eta)= & F_{a}^{(1)}(k ; \eta) P_{0}(k) F_{b}^{(1)}(k ; \eta), \\
P_{a b}^{1-\text { loop }}(k, \eta)= & \int_{\mathbf{q}} P_{0}(q)\left[3 F_{a}^{(1)}(k ; \eta) P_{0}(k) F_{b}^{(3)}(\mathbf{k}, \mathbf{q},-\mathbf{q} ; \eta)\right. \\
& +3 F_{a}^{(3)}(\mathbf{k}, \mathbf{q},-\mathbf{q} ; \eta) P_{0}(k) F_{b}^{(1)}(k ; \eta) \\
& \left.+2 F_{a}^{(2)}(\mathbf{k}-\mathbf{q}, \mathbf{q} ; \eta) P_{0}(|\mathbf{k}-\mathbf{q}|) F_{b}^{(2)}(\mathbf{k}-\mathbf{q}, \mathbf{q} ; \eta)\right], \\
P_{a b}^{2-\text { loop }}(k, \eta)= & \int_{\mathbf{q}, \mathbf{p}} P_{0}(q) P_{0}(p)\left[15 F_{a}^{(1)}(k ; \eta) P_{0}(k) F_{b}^{(5)}(\mathbf{k}, \mathbf{p},-\mathbf{p}, \mathbf{q},-\mathbf{q} ; \eta)\right. \\
& +15 F_{a}^{(5)}(\mathbf{k}, \mathbf{p},-\mathbf{p}, \mathbf{q},-\mathbf{q} ; \eta) P_{0}(k) F_{b}^{(1)}(k ; \eta) \\
& +12 F_{a}^{(2)}(\mathbf{k}-\mathbf{q}, \mathbf{q} ; \eta) P_{0}(|\mathbf{k}-\mathbf{q}|) F_{b}^{(4)}(\mathbf{k}-\mathbf{q}, \mathbf{q}, \mathbf{p},-\mathbf{p} ; \eta) \\
& +12 F_{a}^{(4)}(\mathbf{k}-\mathbf{q}, \mathbf{q}, \mathbf{p},-\mathbf{p} ; \eta) P_{0}(|\mathbf{k}-\mathbf{q}|) F_{b}^{(2)}(\mathbf{k}-\mathbf{q}, \mathbf{q} ; \eta) \\
& +9 F_{a}^{(3)}(\mathbf{k}, \mathbf{q},-\mathbf{q} ; \eta) P_{0}(k) F_{b}^{(3)}(\mathbf{k}, \mathbf{p},-\mathbf{p} ; \eta) \\
& \left.+6 F_{a}^{(3)}(\mathbf{k}-\mathbf{p}-\mathbf{q}, \mathbf{p}, \mathbf{q} ; \eta) P_{0}(|\mathbf{k}-\mathbf{p}-\mathbf{q}|) F_{b}^{(3)}(\mathbf{k}-\mathbf{p}-\mathbf{q}, \mathbf{p}, \mathbf{q} ; \eta)\right] .
\end{aligned}
$$

These expressions are analogous to the familiar SPT loop integrals, except that here the generalized time-dependent kernels $F_{a}^{(n)}$ appear. Note that the indices $a, b$ label the components of $\psi_{a}$, such that for example $P_{11}$ denotes the density auto power spectrum, $P_{22}$ the velocity divergence spectrum, and $P_{12}$ the cross spectrum. In case of multiple fluids, $P_{13}$ is the cross power spectrum between the density contrast of the first and second component, etc. We stress that these indices are unrelated to the conventional naming of the various summands in the 1- and 2-loop integrals within SPT according to the perturbative order of the kernels. Furthermore, we emphasize that, in general, even the linear evolution can be non-trivial, being captured by the transfer functions $F_{a}^{(1)}(k ; \eta)$.

For numerical evaluation, we further modify the expressions inside the square brackets to ensure that large cancellations that occur when one of the arguments of $P_{0}$ goes to zero are accounted for already at the integrand level [39, 40,42]. We then compute the loop integrals using the Suave routine of the Monte-Carlo integration package CUBA [43]. The numerical algorithm at 2-loop is an extended version compared to [39-41], and can be summarized as follows:

1. The Monte-Carlo loop integrator calls the routine computing the integrand for a given set of wavenumbers $\mathbf{p}, \mathbf{q}$. The external wavevector is fixed to $\mathbf{k}=(0,0, k)$ (without loss of generality).

2. All vertices $\gamma_{a b c}\left(\mathbf{k}_{12}, \mathbf{k}_{1}, \mathbf{k}_{2}\right)$ are computed and stored for all sets of possible wavevectors $\mathbf{k}_{1}$ and $\mathbf{k}_{2}$ of the form

$$
\epsilon_{0} \mathbf{k}+\epsilon_{1} \mathbf{p}+\epsilon_{2} \mathbf{q}, \quad \epsilon_{0}=0,1, \epsilon_{1,2}=0, \pm 1 .
$$

3. For each kernel $F_{a}^{(n)}$, evaluated with the particular set of wavevectors occurring in the loop integrand, we solve the set of ordinary differential equations (2.13) numerically. This requires to solve also for the lower order kernels $F_{a}^{(m)}$ and $F_{a}^{(n-m)}$ with $m=$ 
$1, \ldots, n-1$ evaluated on a subset of wavevectors, which can be done recursively. We temporarily store the time-dependent kernels on a sufficiently dense grid between $\eta_{\text {ini }}$ and the output time. This leads to a significant reduction in computing time due to the recursive structure of Eq. (2.13) and since only a limited number of combinations of wavevectors can occur as an argument. We describe the implementation of initial conditions for the kernels in the sections below.

Let us finally comment on an alternative scheme that can capture a non-trivial timedependence, known as time-dependent renormalization group (TRG) [44], which is based on solving coupled equations for the power- and bispectrum. As shown in [45], this scheme can be considered being equivalent to a 1-loop computation of the power spectrum that allows to take an arbitrary time-dependence of $\Omega_{a b}$ into account. Extending this formalism to 2loop would require to include also the trispectrum, which represents significant technical challenges [46]. In addition, even when neglecting the trispectrum, the practical feasibility of the algorithm depends on being able to reduce the computation to a certain moment of the bispectrum. However, this is no longer possible when $\Omega_{a b}$ depends also on wavenumber, as is the case when including an effective viscosity and sound velocity, and also for massive neutrinos. In addition, for an $N$-fluid scheme, the TRG approach would require to track the time-dependence of all $2 N(2 N-1)$ power spectra, while for the approach pursued here it is sufficient to compute the $2 N$ kernels $F_{a}^{(n)}$. Note that the algorithm presented here can be extended to the 1-loop bispectrum with exact time- and scale-dependence [47].

\section{$3 \quad$ Validity of EdS approximation}

In this section we turn to our first application: relaxing the commonly used EdS approximation and computing the matter power spectrum for the first time at 2-loop from kernels with exact time-dependence. The effect of the EdS approximation on the 1-loop correction to the power spectrum has been studied previously in the literature [48-52], where analytic expressions for the relevant time-dependent kernels in generalized cosmologies have been derived. It has been shown that the 1-loop term in the EdS approximation is accurate to less than $1 \%$ at $z=0$ in the mildly non-linear regime. We find that adding the 2-loop correction, the inaccuracy increases to more than $1 \%$ at $k=0.2 \mathrm{~h} / \mathrm{Mpc}$. The approximation works better at earlier times, and indeed we find only a few permille deviation on the relevant scales at $z=0.5$. Future low-redshift surveys may achieve a sensitivity close to this mark, hence it is worth studying the quantitative effects of the EdS approximation on cosmological observables.

Our analysis is based on a $\Lambda$ CDM cosmology with $h=0.6756, \Omega_{\mathrm{b}}=0.04828, \Omega_{\mathrm{cdm}}=$ $0.2638, n_{s}=0.9619$ and $A_{s}=2.215 \cdot 10^{-9}$. The input linear power spectrum as well as the ratio $\Omega_{m} / f^{2}$ is taken from the Boltzmann solver CLASS. As described above, to compute the 1- and 2-loop power spectrum, we solve Eq. (2.13) numerically for each kernel and set of wavenumbers needed in the Monte-Carlo integration. We initialize the time evolution deep inside the matter dominated era (specifically, $z_{\text {ini }}=25$ ). Since $\Omega_{m} / f^{2}$ is very close to unity at that time, it is possible to use the conventional EdS-SPT kernels as initial conditions. In Appendix A, we compare our numerical 1-loop result with the analytic time-dependent kernels from Ref. [48], finding agreement at the sub-permille level and within the numerical error bar of the Monte Carlo integration. We performed numerous additional convergence checks of the numerical implementation that are discussed in Appendix B. 

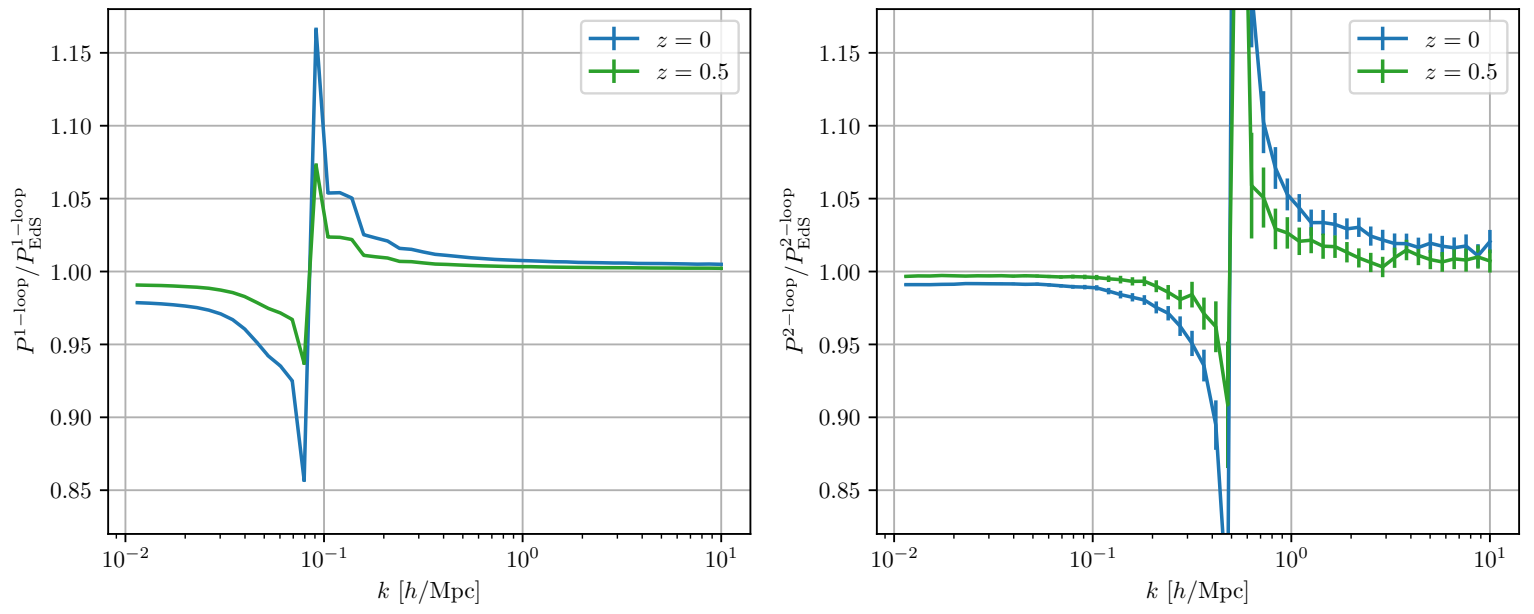

Figure 1. Non-linear corrections to the matter power spectrum with exact time-dependence, normalized to the corresponding terms in the EdS approximation. Left: 1-loop correction and right: 2-loop correction. The blue curves correspond to $z=0$ while the green ones correspond to $z=0.5$. The error bars display the uncertainty from the numerical integration. Note that the spikes at $k \simeq 0.08 h / \mathrm{Mpc}$ (1-loop) and $k \simeq 0.5 h / \mathrm{Mpc}$ (2-loop) are due to the non-linear corrections crossing zero, leading to large relative deviations.
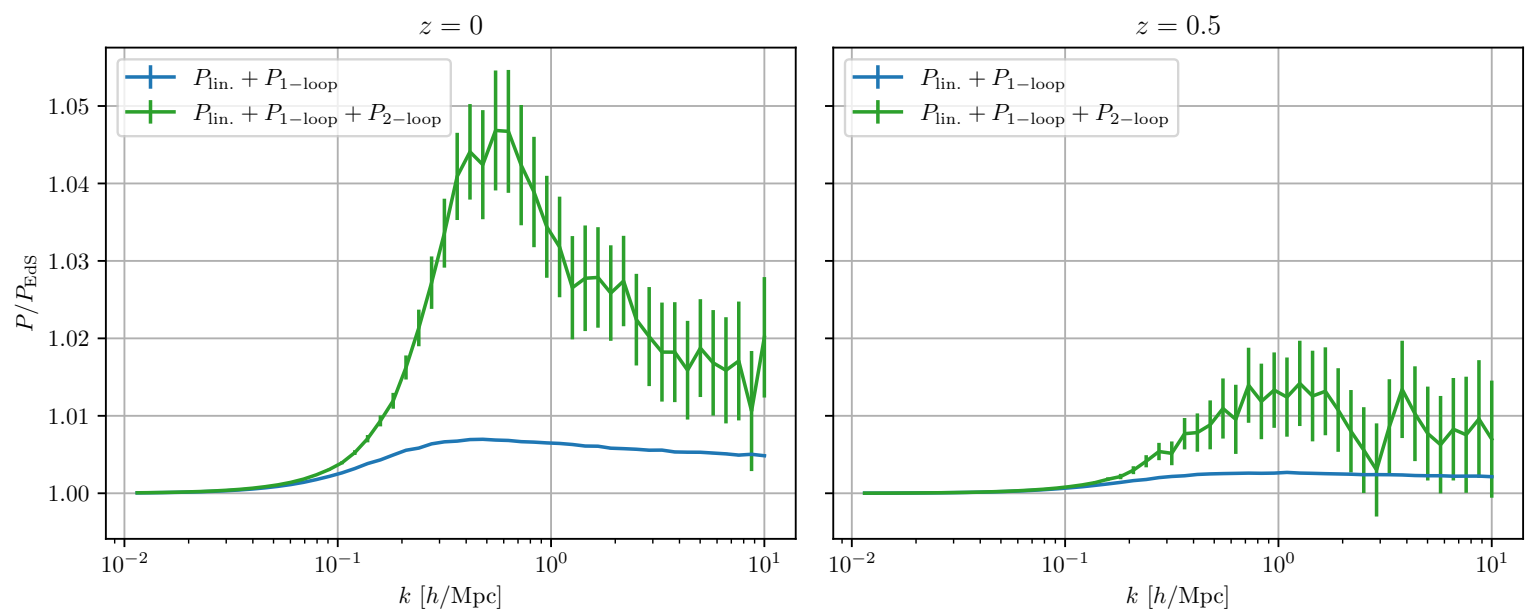

Figure 2. Total matter power spectrum at 1- and 2-loop with exact time-dependence normalized to that in the EdS approximation. Left: Redshift $z=0$. right: $z=0.5$. The uncertainty from the numerical integration is indicated with error bars.

We compute the 1- and 2-loop corrections to the power spectrum both in the EdS approximation and with the exact time-dependence and compare the results. In Fig. 1, this comparison is shown for each loop correction separately and at redshifts $z=0$ and $z=0.5$, respectively. At $z=0.5$ the EdS approximation works better, since the exact solution only very recently starts to deviate from the EdS one. At $k \simeq 0.08 h / \mathrm{Mpc}$, the 1-loop correction switches sign and crosses zero, which yield large relative deviations (similarly for the 2-loop term at $k \simeq 0.5 h / \mathrm{Mpc}$ ). Fig. 2 displays the total power spectrum at 1- and 2-loop normalized to the EdS solution, computed at two redshifts $z=0$ and $z=0.5$. On large scales the linear contribution dominates, which is not affected by the EdS approximation, hence the departure 
from EdS starts to appear around $k \simeq 0.1 \mathrm{~h} / \mathrm{Mpc}$. In addition to the EdS approximation being more reliable at earlier times, the non-linear corrections are smaller compared to the linear power spectrum at $z=0.5$, hence the deviation of the total power spectrum is only a few permille in the weakly non-linear regime of the right plot.

\section{Two-fluid model: CDM + baryons and massive neutrinos}

In this section we set up a two-component fluid model for baryons, CDM and massive neutrinos, and discuss its validity. Baryons and CDM are treated equally as a single component, forming a perfect, pressureless fluid. They interact through gravity with the neutrinos, which are described by a fluid with an effective sound velocity. A fluid model for neutrinos was thoroughly studied in Ref. [53], which found that the fluid description was only accurate at the level of 10-20\%. We aim for far better accuracy than that, hence we follow Ref. [33] and use a hybrid scheme: at high redshift we use the full Boltzmann hierarchy while at low redshift we switch to the two-fluid model. The reasoning behind this is two-fold. Firstly, after the neutrinos become non-relativistic, the coupling between lower and higher moments of the Boltzmann hierarchy is suppressed by powers of $T_{\nu} / m_{\nu}$. This decoupling allows us to follow only the lowest moments at late times. Secondly, non-linear corrections become important at late redshift $z \lesssim 10$, and computing them numerically is manageable in the two-fluid model. Therefore, we match the Boltzmann solution onto the two-fluid model at $z_{\text {match }}=25$, which for the neutrino masses we consider is well after the non-relativistic transition given by

$$
z_{\mathrm{nr}} \simeq 1890 \frac{m_{\nu}}{1 \mathrm{eV}}
$$

We find that depending on the specific expression used for the neutrino effective sound velocity, the two-fluid model agrees with the full Boltzmann solution at (sub-)permille and percent level for the CDM+baryons and neutrino transfer functions, respectively (see below).

We consider four models with neutrino masses $\sum m_{\nu}=0.06,0.15,0.21$ and $0.3 \mathrm{eV}$, respectively. In all models we have three degenerate neutrino species, described jointly by a single fluid component. In principle it is possible to extend the formalism to a multi-fluid where each neutrino species has an individual effective sound velocity. However, cosmological probes are quite insensitive to the specific details of neutrino physics except for the absolute mass scale, and the error introduced by using degenerate neutrino species appears to be negligible compared to expected sensitivity of future surveys, see e.g. Ref. [54].

Neglecting vorticity and dropping non-linear terms for the moment, the continuity and Euler equations for the $\mathrm{CDM}+$ baryons and neutrino density contrast and velocity divergence are

$$
\begin{aligned}
\partial_{\tau} \delta_{\mathrm{cb}}+\theta_{\mathrm{cb}} & =0, \\
\partial_{\tau} \theta_{\mathrm{cb}}+\mathcal{H} \theta_{\mathrm{cb}}+\frac{3}{2} \mathcal{H}^{2} \Omega_{m}\left[f_{\nu} \delta_{\nu}+\left(1-f_{\nu}\right) \delta_{\mathrm{cb}}\right] & =0, \\
\partial_{\tau} \delta_{\nu}+\theta_{\nu} & =0, \\
\partial_{\tau} \theta_{\nu}+\mathcal{H} \theta_{\nu}+\frac{3}{2} \mathcal{H}^{2} \Omega_{m}\left[f_{\nu} \delta_{\nu}+\left(1-f_{\nu}\right) \delta_{\mathrm{cb}}\right]-k^{2} c_{s}^{2} \delta_{\nu}+k^{2} \sigma & =0 .
\end{aligned}
$$

Here, the combined baryon and CDM density contrast is

$$
\delta_{\mathrm{cb}}=\frac{f_{\mathrm{b}} \delta_{\mathrm{b}}+f_{\mathrm{cdm}} \delta_{\mathrm{cdm}}}{f_{\mathrm{b}}+f_{\mathrm{cdm}}}
$$


where the energy density fraction for each species is $f_{i} \equiv \Omega_{i} / \Omega_{m}$. The velocity divergence $\theta_{\mathrm{cb}}$ is defined equivalently. The energy density fraction of neutrinos is constant for $z \ll z_{\mathrm{nr}}$,

$$
f_{\nu}=\frac{1}{\Omega_{m}(z=0)} \frac{\sum m_{\nu}}{93.14 h^{2} \mathrm{eV}} .
$$

Specifically, this gives $f_{\nu}=0.0045,0.0112,0.0156$, and 0.0221 for $\sum m_{\nu}=0.06,0.15,0.21$ and $0.3 \mathrm{eV}$, respectively. For the lowest neutrino masses, the exact neutrino fraction $f_{\nu}(z)$ differs about $1 \%$ around $z_{\text {match }}=25$ from the constant approximation above, however taking the exact time-dependence into account only alters the transfer functions at $z=0$ at the order of $10^{-4}$, hence we neglect it.

Lastly, in the Euler equation for the neutrino component, Eq. (4.2d), the sound velocity term expresses the free-streaming of the neutrinos: $c_{s}^{2}=\delta P_{\nu} / \delta \rho_{\nu}$. In addition, $\sigma$ denotes the anisotropic stress. Both $c_{s}^{2}$ and $\sigma$ needs to be specified in order to close the system in Eqs. (4.2), and we return to this issue below.

Again, we find it convenient to rewrite the continuity and Euler equations in terms of $\eta=$ $\ln D(z)$. However, for a model with massive neutrinos the growth factor is $k$-dependent, which complicates the analysis. To avoid this issue, we instead use the growth factor $D_{f_{\nu}=0}(z)$ of a corresponding reference cosmology with massless neutrinos. Specifically, given a cosmology with massive neutrinos, the reference cosmology has the exact same parameters except for the CDM energy density which is adjusted such that the total matter energy densities of the models are the same. The equation for the growth factor $D_{f_{\nu}=0}(z)$ is

$$
\frac{\mathrm{d}^{2} D_{f_{\nu}=0}(z)}{\mathrm{d} \ln a^{2}}+\left(1+\frac{\mathrm{d} \ln \mathcal{H}}{\mathrm{d} \ln a}\right) \frac{\mathrm{d} D_{f_{\nu}=0}(z)}{\mathrm{d} \ln a}-\frac{3}{2} \Omega_{m} D_{f_{\nu}=0}(z)=0 .
$$

For scales much larger than the neutrino free-streaming scale, neutrinos behave as CDM and the growth factors of the two models agree. We define the tuple $\psi_{a}$ as

$$
\psi_{1}=\delta_{\mathrm{cb}}, \quad \psi_{2}=-\frac{\theta_{\mathrm{cb}}}{\mathcal{H} f}, \quad \psi_{3}=\delta_{\nu}, \quad \psi_{4}=-\frac{\theta_{\nu}}{\mathcal{H} f},
$$

where $f=\mathrm{d} \ln D_{f_{\nu}=0} / \mathrm{d} \ln a$. Using $\partial_{\tau}=\mathcal{H} f \partial_{\eta}$, the linearized continuity and Euler equations become

$$
\partial_{\eta} \psi_{a}+\Omega(k, \eta)_{a b} \psi_{b}=0
$$

with

$$
\Omega(k, \eta)=\left(\begin{array}{cccc}
0 & -1 & 0 & 0 \\
-\frac{3}{2} \frac{\Omega_{m}}{f^{2}}\left(1-f_{\nu}\right) & \frac{3}{2} \frac{\Omega_{m}}{f^{2}}-1 & -\frac{3}{2} \frac{\Omega_{m}}{f^{2}} f_{\nu} & 0 \\
0 & 0 & 0 & -1 \\
-\frac{3}{2} \frac{\Omega_{m}}{f^{2}}\left(1-f_{\nu}\right) & 0 & -\frac{3}{2} \frac{\Omega_{m}}{f^{2}}\left[f_{\nu}-k^{2} c_{s, \mathrm{eff}}^{2}(k, \eta)\right] & \frac{3}{2} \frac{\Omega_{m}}{f^{2}}-1
\end{array}\right),
$$

where $c_{s, \text { eff }}^{2}$ is an effective sound velocity that will be discussed shortly. In solving Eq. (4.6), we write $\psi_{a}(\mathbf{k}, \eta)=F_{a}^{(1)}(k, \eta) \delta_{\mathrm{cb}}\left(\mathbf{k}, \eta_{\text {match }}\right)$ and use the growing mode eigenvector of the matrix $\Omega\left(k, \eta_{\text {match }}\right)$ as initial condition for the linear kernels $F_{a}^{(1)}$ (suitably normalized so that $\left.F_{1}^{(1)}\left(k, \eta_{\text {match }}\right)=1\right)$. We will clarify the reasoning for this choice of initial conditions when we move on to non-linear corrections in the next section. Thus, we may compute e.g. the linear CDM+baryons and neutrino cross power spectrum at some time $\eta$ by

$$
P_{\mathrm{cb}, \nu}(k, \eta)=F_{1}^{(1)}(k, \eta) F_{3}^{(1)}(k, \eta) P_{\mathrm{cb}, \mathrm{cb}}\left(k, \eta_{\text {match }}\right),
$$

where $P_{\mathrm{cb}, \mathrm{cb}}\left(k, \eta_{\mathrm{match}}\right)$ is taken from CLASS. 


\subsection{Sound velocity and anisotropic stress}

We will now describe two ways to close the system in Eq. (4.6): (i) by approximating the sound velocity by the adiabatic sound velocity and neglecting the anisotropic stress and (ii) by integrating the Boltzmann equation for neutrinos numerically to obtain the exact sound velocity and anisotropic stress. Both methods will be used and compared when we discuss non-linear corrections in the next section.

Adiabatic approximation (2F-ad) In the non-relativistic limit the sound velocity of the neutrinos approaches the adiabatic sound velocity $c_{g}$, which can be related to the velocity dispersion $\sigma_{\nu}$ (assuming $\sigma_{\nu}^{2} \ll 1$ ) [53]:

$$
c_{s}^{2} \equiv \frac{\delta P}{\delta \rho} \underset{z \ll z_{\mathrm{nr}}}{\longrightarrow} c_{g}^{2}=\frac{5}{9} \sigma_{\nu}^{2}=\frac{25}{3} \frac{\zeta(5)}{\zeta(3)}\left(\frac{T_{\nu}}{m_{\nu}}\right)^{2} .
$$

Assuming also that the anisotropic stress can be neglected in this limit, the effective sound velocity entering the two-fluid equations in Eqs. (4.6) and (4.7) is

$$
c_{s, \mathrm{eff}}^{2}=\frac{2}{3 \Omega_{m} \mathcal{H}^{2}} c_{g}^{2} \simeq \frac{1.214}{\Omega_{m}(z=0)}\left(\frac{1 \mathrm{eV}}{m_{\nu}}\right)^{2}(1+z) \frac{\mathrm{Mpc}^{2}}{h^{2}} .
$$

We will refer to this scheme in the future with the label $2 \mathrm{~F}$-ad. It has the benefit of being simpler to implement in practice compared to the more accurate scheme that we turn to next.

Exact effective sound velocity (2F) The idea of this scheme is to obtain an optimal fluid description that is informed about the impact of the linear perturbations to the complete neutrino distribution function, including all of its higher moments. This is realized by using the exact neutrino sound velocity and anisotropic stress in the Euler equation, derived from a full solution of the linearized collision-less Boltzmann equation [55]

$$
\left(\partial_{\tau}+\frac{\mathrm{i} q}{\epsilon(q, \tau)}(\mathbf{k} \cdot \hat{\mathbf{n}})\right) \Psi(\mathbf{k}, \hat{\mathbf{n}}, q, \tau)+\frac{\mathrm{d} \ln f_{0}}{\mathrm{~d} \ln q}\left(\partial_{\tau} \phi(k, \tau)-\frac{\mathrm{i} \epsilon(q, \tau)}{q}(\mathbf{k} \cdot \hat{\mathbf{n}}) \psi(k, \tau)\right)=0 .
$$

Here, $\Psi$ is the linear perturbation to the neutrino distribution function, $f=f_{0}(1+\Psi)$, $\hat{\mathbf{n}}=\mathbf{q} / q$ and $\phi$ and $\psi$ are metric perturbations in the conformal Newtonian gauge. Furthermore, $q$ and $\epsilon(q, \tau)=\sqrt{q^{2}+a^{2}(\tau) m^{2}}$ are the comoving momentum and energy of a particle, respectively. Neutrinos decoupled while they were ultra-relativistic, hence the unperturbed distribution function $f_{0}$ remains in the form of an ultra-relativistic Fermi-Dirac distribution also after the non-relativistic transition. Integrating Eq. (4.11) and projecting onto Legendre polynomials $P_{l}(\mathbf{k} \cdot \hat{\mathbf{n}} / k)$ yields the following multipole solutions [53]:

$$
\begin{aligned}
& \Psi_{l}(k, q, \tau)=\frac{\mathrm{d} \ln f_{0}(q)}{\mathrm{d} \ln q} \times\left(\phi\left(k, \tau_{i}\right) j_{l}\left[k y\left(\tau_{i}, \tau\right)\right]-\phi(k, \tau) \delta_{l 0}\right. \\
& \left.-k \int_{\tau_{i}}^{\tau} \mathrm{d} \tilde{\tau}\left[\frac{\epsilon(q, \tilde{\tau})}{q} \psi(k, \tilde{\tau})+\frac{q}{\epsilon(q, \tilde{\tau})} \phi(k, \tilde{\tau})\right]\left[\frac{l}{2 l+1} j_{l-1}[k y(\tilde{\tau}, \tau)]-\frac{l+1}{2 l+1} j_{l+1}[k y(\tilde{\tau}, \tau)]\right]\right) \\
& +\sum_{l^{\prime}} \sum_{l^{\prime \prime}}(-\mathrm{i})^{l^{\prime}+l^{\prime \prime}-l}\left(2 l^{\prime}+1\right)\left(2 l^{\prime \prime}+1\right) \Psi_{l^{\prime}}\left(k, q, \tau_{i}\right) j_{l^{\prime \prime}}\left[k y\left(\tau_{i}, \tau\right)\right]\left(\begin{array}{lll}
l & l^{\prime} & l^{\prime \prime} \\
0 & 0 & 0
\end{array}\right)^{2} \text {, }
\end{aligned}
$$


where $j_{l}$ are spherical Bessel functions, $\left(\begin{array}{lll}l & l^{\prime} & l^{\prime \prime} \\ 0 & 0 & 0\end{array}\right)$ is the Wigner 3-j symbol, and

$$
y\left(\tau_{a}, \tau_{b}\right)=\int_{\tau_{a}}^{\tau_{b}} \mathrm{~d} \tau \frac{q}{\epsilon(q, \tau)} .
$$

Note that we fixed a sign typo from Ref. [53] in the $\phi$-term of the second line of Eq. (4.12).

The perturbations $\Psi_{l}$ are related to the density contrast, velocity divergence and anisotropic stress through integrals over momentum:

$$
\begin{aligned}
\bar{\rho}(\tau) & =\frac{4 \pi}{a^{4}(\tau)} \int \mathrm{d} q q^{2} \epsilon(q, \tau) f_{0}(q), \\
\bar{P}(\tau) & =\frac{4 \pi}{3 a^{4}(\tau)} \int \mathrm{d} q q^{2} \frac{q^{2}}{\epsilon(q, \tau)} f_{0}(q), \\
\delta \rho(k, \tau) & =\frac{4 \pi}{a^{4}(\tau)} \int \mathrm{d} q q^{2} \epsilon(q, \tau) f_{0}(q) \Psi_{0}(k, q, \tau), \\
\delta P(k, \tau) & =\frac{4 \pi}{3 a^{4}(\tau)} \int \mathrm{d} q q^{2} \frac{q^{2}}{\epsilon(q, \tau)} f_{0}(q) \Psi_{0}(k, q, \tau), \\
(\bar{\rho}+\bar{P}) \theta(k, \tau) & =\frac{4 \pi k}{a^{4}(\tau)} \int \mathrm{d} q q^{3} f_{0}(q) \Psi_{1}(k, q, \tau), \\
(\bar{\rho}+\bar{P}) \sigma(k, \tau) & =\frac{8 \pi}{3 a^{4}(\tau)} \int \mathrm{d} q q^{2} \frac{q^{2}}{\epsilon(q, \tau)} f_{0}(q) \Psi_{2}(k, q, \tau) .
\end{aligned}
$$

Thus, using Eqs. (4.12) and (4.14) we may compute the exact sound velocity $c_{s}^{2}(k, \tau) \equiv$ $\delta P / \delta \rho$, density perturbation $\delta(k, \tau) \equiv \delta \rho / \bar{\rho}$ and anisotropic stress $\sigma(k, \tau)$ to obtain the effective sound velocity which is used in the two-fluid evolution,

$$
c_{s, \mathrm{eff}}^{2}=\frac{2}{3 \Omega_{m} \mathcal{H}^{2}}\left(c_{s}^{2}(k, \tau)-\frac{\sigma(k, \tau)}{\delta(k, \tau)}\right) .
$$

In practice, we simplify Eq. (4.12) by assuming $\phi=\psi$ at all times and $\psi=$ const before vacuum energy domination (specifically, before $z=5$ ). In addition, we neglect the contributions from the initial perturbations $\Psi_{l}\left(k, q, \tau_{i}\right)$ since the integral term dominates at late times. The gravitational potential is obtained from a Boltzmann solver (CLASS) and $\tau_{i}=1 \mathrm{Mpc}$ is used. We check explicitly for a subset of wavenumbers and the neutrino masses we consider that dropping these assumptions has negligible impact on the numerical results.

The sound velocity and anisotropic stress may also be extracted from CLASS, which solves the Boltzmann equation (4.11) by immediately projecting onto Legendre polynomials and integrating the resulting hierarchy of differential equations for the $\Psi_{l}$ 's. ${ }^{1}$ A truncation of the hierarchy at some maximum multipole $l_{\max }$ is needed in order to be able to solve it numerically, which may introduce inaccuracies that propagate the hierarchy to lower multipoles. Note that this problem is not present in Eq. (4.12), since there is no coupling between multipoles (up to backreaction on the gravitational potentials). Nevertheless, using Eq. (4.12) for the neutrino perturbation in a Boltzmann solver would be very time-consuming, since the integral has to be performed at every time step in order to update transfer functions and metric perturbations (we avoid this by taking the metric perturbations as external input).

\footnotetext{
${ }^{1}$ In our utilization of CLASS for cosmologies with massive neutrinos, we turn off the default fluid approximation for neutrinos, i.e. we use ncdm_fluid_approximation $=3$ and $1 \_$max $=17$ unless stated otherwise.
} 

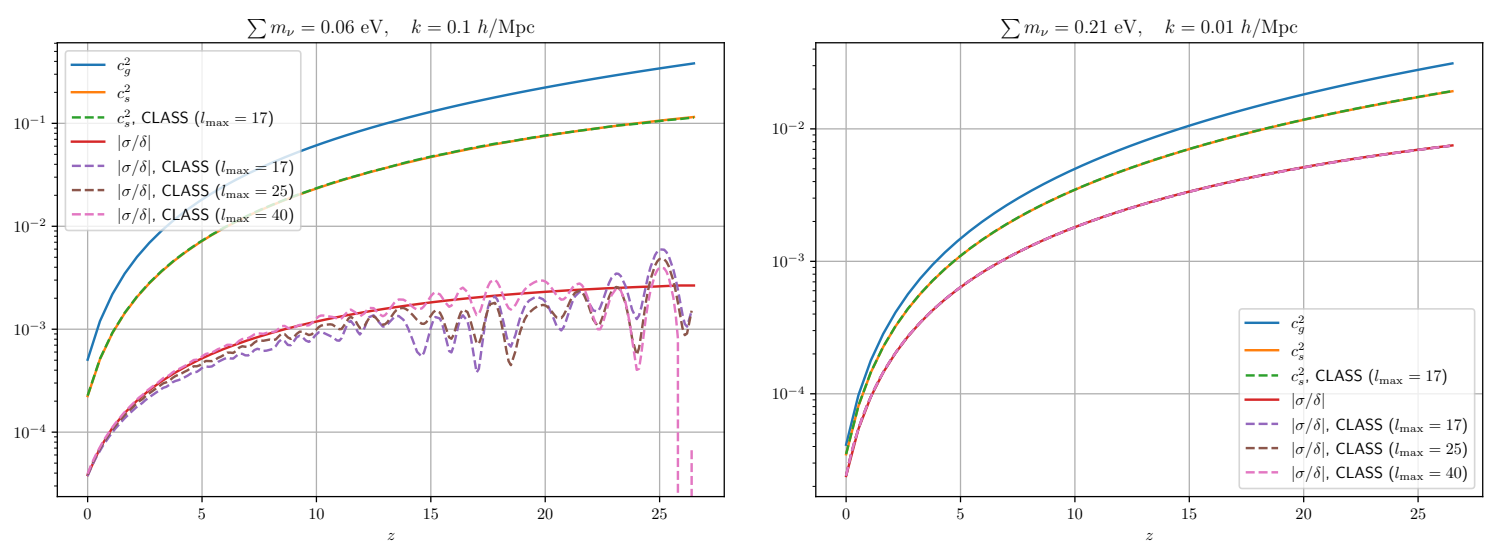

Figure 3. Adiabatic sound velocity $c_{g}^{2}$, sound velocity $c_{s}^{2}$ and absolute value of the anisotropic stress divided by the density contrast, $|\sigma / \delta|$, as a function of redshift from our numerical computation and from CLASS using different maximum number of multipoles $l_{\max }$ (note that the difference is invisible except for $|\sigma / \delta|$ in the left plot). Left: neutrino mass $\sum m_{\nu}=0.06 \mathrm{eV}$ and wavenumber $k=0.1$ h/Mpc. Right: $\sum m_{\nu}=0.21 \mathrm{eV}$ and $k=0.01 \mathrm{~h} / \mathrm{Mpc}$.

In Fig. 3, we compare our numerical results for the sound velocity $c_{s}^{2}$ and anisotropic stress over density contrast $|\sigma / \delta|$ with CLASS for two choices of neutrino mass and wavenumber. In addition, the adiabatic sound velocity $c_{g}^{2}$ is plotted. We see that the sound velocity is in good agreement with CLASS; a closer quantitative analysis yields agreement of around $1 \%$, also for a larger set of wavenumbers and neutrino masses, and independent of the maximum number of multipoles $l_{\max }$ used in CLASS. Furthermore, for high neutrino masses and low wavenumbers, the ratio of anisotropic stress and density contrast also has percent level agreement with that from CLASS. In the other limit, the CLASS solution develops oscillatory behavior, and only the mean value of the oscillations follows our numerical result. This is seen for $k=0.1 h / \mathrm{Mpc}$ in the left plot of Fig. 3. For even smaller scales, the oscillations become more pronounced and results using different multipole truncation number $l_{\max }$ do not converge to the same answer even at late times. Nonetheless, in this limit $|\sigma / \delta|$ is suppressed compared to the sound velocity, so the impact on the velocity divergence in the Euler equation is small. In principle, we could have used CLASS to compute the sound velocity and anisotropic stress entering Eq. (4.15) and in turn entering the two-fluid model, but the oscillatory behavior on small scales makes this impractical, hence we opt for numerically solving Eqs. (4.12) and (4.14).

In the following we will use the label $2 \mathrm{~F}$ for the two-fluid model with the exact effective sound velocity in Eq. (4.15).

\subsection{Comparison with Boltzmann solver}

Having set up the two-fluid model for CDM+baryons and massive neutrinos in the previous subsection, we proceed to compare this scheme at the linear level with a Boltzmann solver (CLASS). The total matter power spectrum is the weighted sum of the CDM+baryons power spectrum, the neutrino power spectrum and the cross power spectrum between CDM+baryons and neutrinos:

$$
P_{\mathrm{m}, \mathrm{m}}=\left(1-f_{\nu}\right)^{2} P_{\mathrm{cb}, \mathrm{cb}}+2\left(1-f_{\nu}\right) f_{\nu} P_{\mathrm{cb}, \nu}+f_{\nu}^{2} P_{\nu, \nu} .
$$




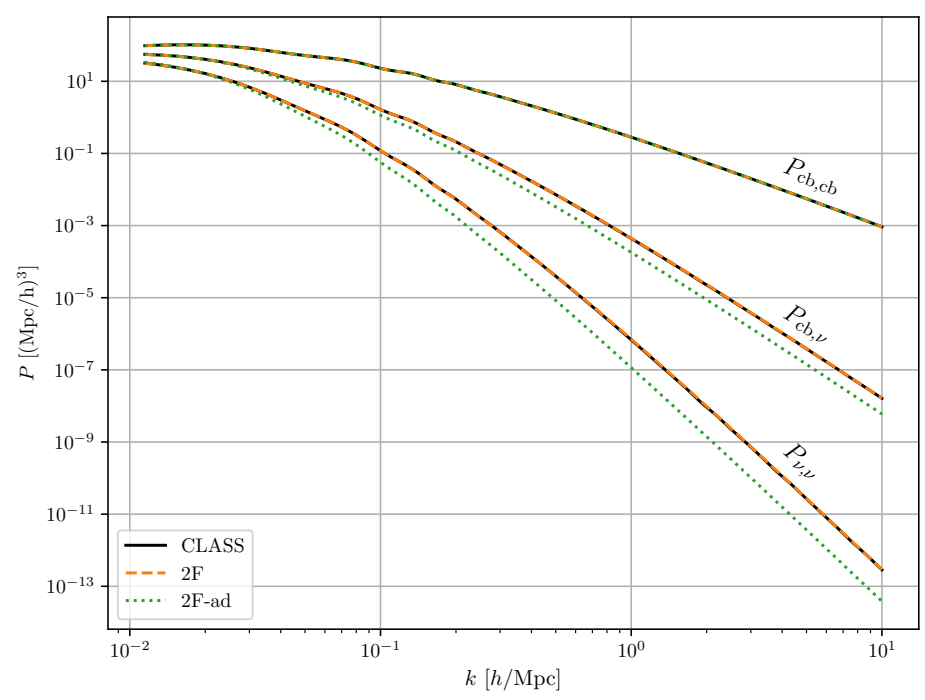

Figure 4. Contributions to the linear matter power spectrum at $z=0$ in a cosmology with $\sum m_{\nu}=0.15 \mathrm{eV}$. The solid black lines correspond to the CLASS solution, the dashed orange lines to the exact two-fluid solution and the dotted green lines to the two-fluid solution using the adiabatic approximation.

The various contributions are shown in Fig. 4 at redshift $z=0$ and for neutrino mass $\sum m_{\nu}=0.15 \mathrm{eV}$, where we include the results from the two-fluid model (both using adiabatic approximation and exact sound velocity) and from CLASS. The neutrino power spectrum and the cross power spectrum are suppressed compared to the CDM+baryons power spectrum due to free-streaming of the neutrinos on scales smaller than the free-streaming scale $k_{\mathrm{FS}}=$ $1 / c_{s, \text { eff }}^{2} \sim 10^{-2} h /$ Mpc. Since in addition $f_{\nu} \ll 1$, the total matter power spectrum is dominated by the $P_{\mathrm{cb}, \mathrm{cb}}$ term on these scales. However, through the backreaction on the gravitational potential, the presence of massive, free-streaming neutrinos leads to a reduction of growth in the baryon and CDM sector, which at the linear level yields the well known $\sim 8 f_{\nu}$ reduction of the total power spectrum. Therefore it is necessary to calculate the neutrino transfer function accurately even when neglecting the last terms of Eq. (4.16).

Fig. 5 displays the relative difference between the two-fluid model and the CLASS solution for the neutrino masses we consider (left: adiabatic approximation, right: exact sound velocity). We see that the two-fluid model with adiabatic approximation underestimates the neutrino-neutrino and cross power spectra by a significant amount (this is also evident from Fig. 4). The decrease in power is caused by the adiabatic approximation overestimating the sound velocity, as is apparent from Fig. 3, leading to suppressed growth on scales smaller than the free-streaming scale. On scales close to and smaller than the free-streaming scale $k_{\mathrm{FS}}$, the Poisson term begins to dominate in the neutrino Euler equation, so the performance of the adiabatic model improves. We note that the adiabatic approximation works better for increasing neutrino masses, where a larger fraction of the neutrino distribution has become sufficiently non-relativistic, and the sound velocity is further in approaching the adiabatic limit. On the other hand, when $f_{\nu}$ is larger the error in the neutrino sector affects the total matter power spectrum to greater extent: the gravitational coupling between CDM+baryons and neutrinos is larger and the last two terms in Eq. (4.16) are more important. Nevertheless, for the total power spectrum and the neutrino masses we consider, we find agreement 

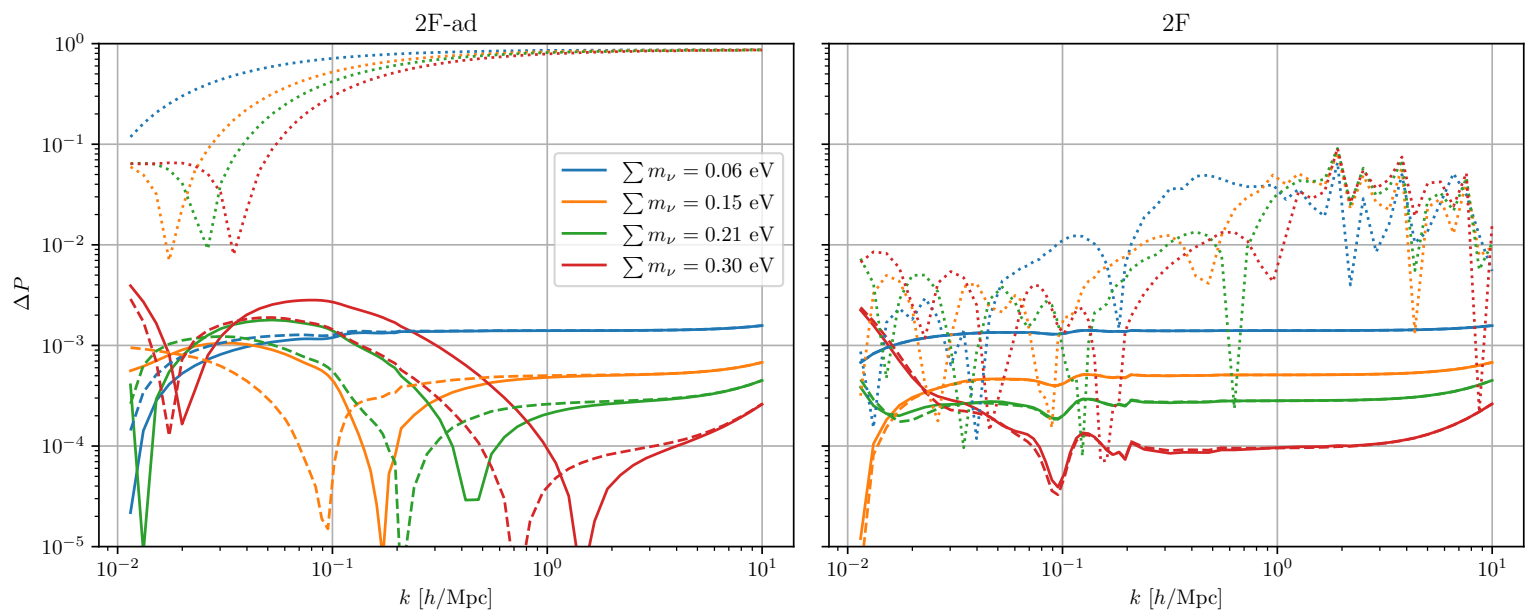

Figure 5. Relative difference between the linear power spectrum computed in the two-fluid model and the Boltzmann solution, $\Delta P=\left|P^{\text {Two-fluid }} / P^{\text {Boltzmann }}-1\right|$, for various neutrino masses at $z=0$. The solid lines correspond to the total matter power spectrum $\Delta P_{\mathrm{m}, \mathrm{m}}$, the dashed lines correspond to the CDM+baryons power spectrum $\Delta P_{\mathrm{cb}, \mathrm{cb}}$ and the dotted lines correspond to the neutrino power spectrum $\Delta P_{\nu, \nu}$. Left: Two-fluid model using the adiabatic sound velocity approximation (scheme 2F-ad). Right: Two-fluid model using exact sound velocity and anisotropic stress (scheme 2F).

between the adiabatic model and the CLASS solution of a few permille.

As expected, the two-fluid model with exact effective sound velocity agrees excellently with the Boltzmann solution, with deviations of the order of $10^{-4}-10^{-3}$ depending on the neutrino mass, as seen in the right plot of Fig. 5 . The neutrino power spectrum agrees with the Boltzmann solution from CLASS at percent level or better for $k \sim 0.1 \mathrm{~h} / \mathrm{Mpc}$, and at the few percent level for $k \gtrsim 1 h / \mathrm{Mpc}$. However in the latter region the Boltzmann solution from CLASS also differs by a few percent depending on the maximum number of multipoles $l_{\max }$ included in the Boltzmann hierarchy, indicating that inaccuracies due to the truncation of the hierarchy propagate to lower multipoles.

Finally, we comment on a few alternative approximations for neutrinos that truncate the Boltzmann hierarchy at $l_{\max }=2$, and hence include an evolution equation for the anisotropic stress in the fluid equations. Various trunction schemes have been used for $\Psi_{3}$ to close the system: the simplest one is $\Psi_{3}=0$ [53], but this is rather inaccurate and the errors propagate to lower multipoles. An improved version is to use the spherical Bessel function recurrence relation [55], and a similar scheme is used by the default neutrino treatment in CLASS [56]. Nevertheless, the neutrino power spectrum is reduced by a factor $\mathcal{O}(10 \%)$ around $k=0.1 \mathrm{~h} / \mathrm{Mpc}$ using this truncation scheme, compared to using the full Boltzmann hierarchy. It was noted in Ref. [57] that due to the gravitational source terms, the Bessel relation poorly captures the behavior of $\Psi_{3}$, and using instead an empirical formula yields percent agreement for the neutrino power spectrum. Our two-fluid model goes even further in that it captures the effect of all higher order cumulants effectively through the sound velocity and anisotropic stress in the Euler equation. In this sense, the agreement with the full Boltzmann hierarchy is largely a consistency check: the only difference between the Boltzmann equations and the $2 \mathrm{~F}$ scheme is the neglection of the neutrino equation of state in the latter.

We conclude that the two-fluid model with neutrino sound velocity and anisotropic 
stress computed from an exact solution of the linear Boltzmann equation works at the $10^{-4}$ to $10^{-3}$ level for the linear total matter power spectrum on scales $k \lesssim 1 \mathrm{~h} / \mathrm{Mpc}$, making it suitable for a precision comparison at higher order in perturbation theory.

\section{Non-linear power spectrum with massive neutrinos}

In this section we compute non-linear corrections to the matter power spectrum in the presence of massive neutrinos. We use the two-fluid model described in the previous section, and compare to other approximation schemes used in the previous literature.

We use the algorithm presented in Sec. 2.2 to compute the 1- and 2-loop power spectrum with exact time- and scale-dependence, and specify some details specific to massive neutrinos in the following. Including non-linear terms in the two-fluid equations in Eq. (4.6) gives an equation of motion of the form of Eq. (2.10) where the non-zero vertices are

$$
\begin{aligned}
& \gamma_{121}\left(\mathbf{k}, \mathbf{k}_{1}, \mathbf{k}_{2}\right)=\gamma_{343}\left(\mathbf{k}, \mathbf{k}_{1}, \mathbf{k}_{2}\right) \equiv \alpha\left(\mathbf{k}_{1}, \mathbf{k}_{2}\right), \\
& \gamma_{222}\left(\mathbf{k}, \mathbf{k}_{1}, \mathbf{k}_{2}\right)=\gamma_{444}\left(\mathbf{k}, \mathbf{k}_{1}, \mathbf{k}_{2}\right) \equiv \beta\left(\mathbf{k}_{1}, \mathbf{k}_{2}\right) .
\end{aligned}
$$

We expand the density contrast and velocity divergence of the CDM+baryon and neutrino components perturbatively according to Eq. (2.12). Note that the linear evolution matrix $\Omega_{a b}(k, \eta)$ is both time- and scale-dependent due to the neutrino effective sound velocity.

To solve the differential equation (2.13), we also need an initial condition for the kernels at $\eta_{\text {match }}$. A simple initial condition setup would be to extend what we did in the EdS analysis and use EdS-SPT and 0 as initial conditions for the CDM+baryons and neutrino kernels, respectively. The reasoning behind this would be that $\eta_{\text {match }}$ is deep in the matter dominated era, where the EdS approximation is accurate, and the neutrino density contrast and velocity divergence are much smaller than the CDM+baryons density contrast in most of the integration domain, so that $F_{3}^{(n)}, F_{4}^{(n)} \ll 1$. However, this works poorly in practice since the initial condition excites transient solutions that propagate the kernel hierarchy and do no entirely decay away by $\eta=0(z=0)$. In particular, the EdS linear growing mode solution $(1,1)$ (which is also the linear growing mode solution of Eq. (2.3)) is not the linear growing mode solution of Eq. (4.6). Rather, the first two components of the eigenvector of $\Omega(k, \eta)$ in Eq. (4.7) corresponding to the growing mode differs by a factor $\mathcal{O}(0.01)$ to $(1,1)$ depending on $\eta$ and $k$.

Having this in mind, given the growing mode eigenvector $u_{a}(k)$ of $\Omega\left(k, \eta_{\text {match }}\right)$, we set $F_{a}^{(1)}\left(k, \eta_{\text {match }}\right)=u_{a}(k) / u_{1}(k)$ as initial condition for the linear kernels. Note that the normalization implies $F_{1}^{(1)}\left(k, \eta_{\text {match }}\right)=1$, consistent with the convention adopted in Sec. 4 , implying $\delta_{0}(\mathbf{k})=\delta_{\mathrm{cb}}^{(1)}\left(\mathbf{k}, \eta_{\text {match }}\right)$ in Eq. (2.12). Initializing the higher order kernels in the growing mode is more tricky. In principle, it is possible to analytically solve Eq. (2.13) with $\Omega=\Omega\left(k, \eta_{\text {match }}\right)$ time-independent, obtaining a recursion solution similar to that in EdS-SPT with a dependence on $k$. Instead, we let the system of differential equations in the numerics reach the growing mode by extrapolating far back in time: starting at $\eta_{\text {asymp }}=-10$ (corresponding to $z \sim 10^{4}$ ) we set

$$
F_{a}^{(n)}\left(k, \eta_{\text {asymp }}\right)= \begin{cases}\frac{u_{a}(k)}{u_{1}(k)} \mathrm{e}^{\lambda_{1}\left(\eta_{\text {asymp }}-\eta_{\text {match }}\right)}, & n=1, \\ 0, & n>1,\end{cases}
$$

where $\lambda_{1}$ is the growing mode eigenvalue of $\Omega\left(k, \eta_{\text {match }}\right)$, and evolve the kernels to $\eta_{\text {match }}$ using $\Omega=\Omega\left(k, \eta_{\text {match }}\right)$. This way, the linear kernels remain in the growing mode solution (by 


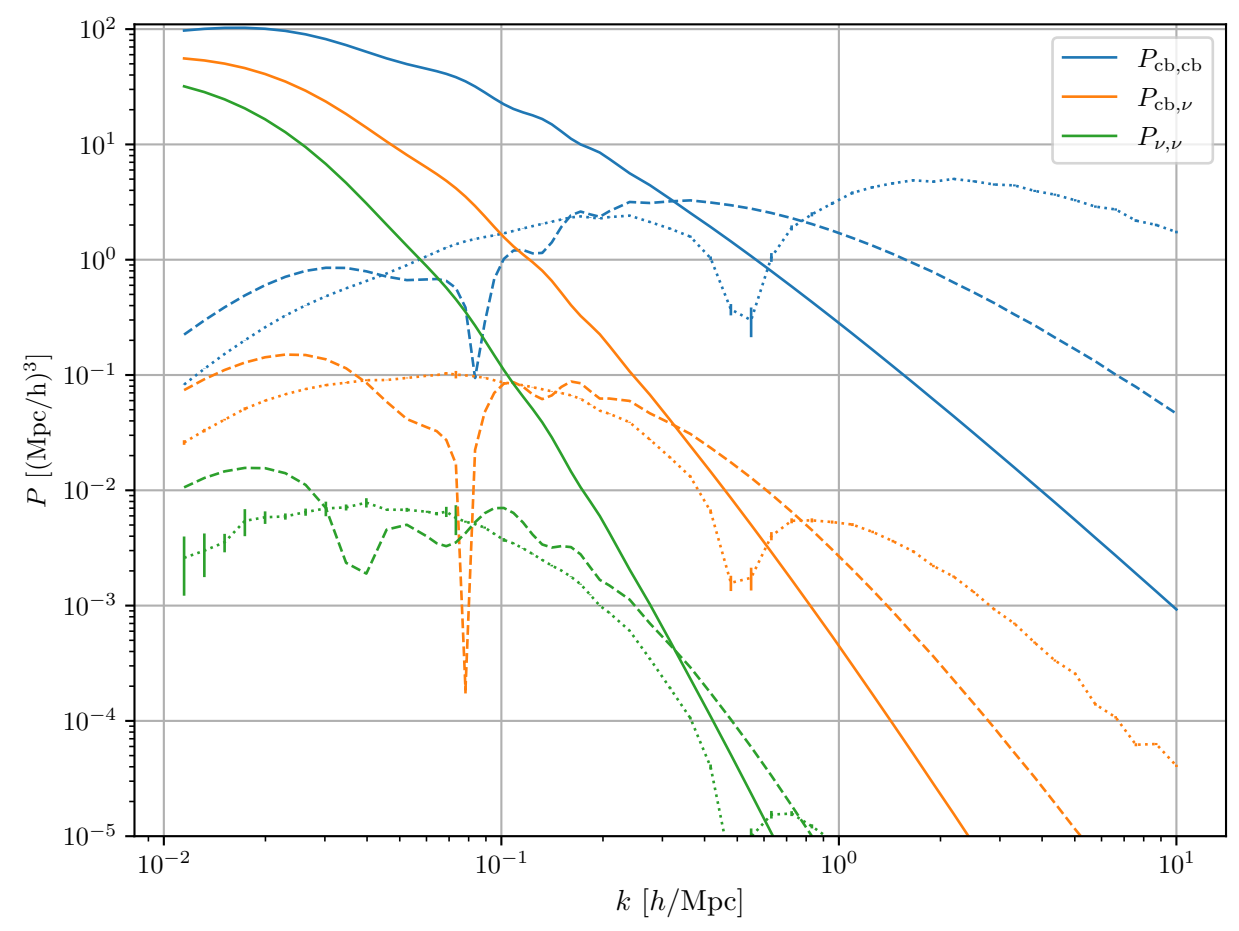

Figure 6. Contributions to the matter power spectrum at $z=0$ computed in the $2 \mathrm{~F}$ scheme for neutrino mass $\sum m_{\nu}=0.15 \mathrm{eV}$. Solid lines correspond to the linear contribution, while dashed and dotted lines correspond to the absolute value of the 1- and 2-loop corrections, respectively. Error bars (only visible in a few places) indicate uncertainty from the numerical integration.

construction) and any transient modes in the higher order kernels have decayed by $\eta_{\text {match }}$. Thus, the entire kernel hierarchy resides in the growing mode of $\Omega\left(k, \eta_{\text {match }}\right)$ when we turn on dynamics for $\eta>\eta_{\text {match }}$. Note that a similar strategy was used in the 1-loop analysis of [33] to avoid transients that occur within the TRG framework [45].

The numerical analysis is based on a $\Lambda \mathrm{CDM}$ cosmology with massive neutrinos and otherwise the same parameters as in Sec. 3: $h=0.6756, \Omega_{\mathrm{b}}=0.04828, \Omega_{\mathrm{cdm}}=0.2638$, $n_{s}=0.9619$ and $A_{s}=2.215 \cdot 10^{-9}$. We take the input linear CDM+baryons power spectrum at $z_{\text {match }}=25$ and the ratio $\Omega_{m} / f^{2}$ from CLASS. Fig. 6 shows the various contributions to the matter power spectrum at 2-loop with neutrino mass $\sum m_{\nu}=0.15 \mathrm{eV}$, computed in the two-fluid model with exact sound velocity (scheme $2 \mathrm{~F}$ ). As one would expect, the neutrino-neutrino and CDM+baryons-neutrino cross correlation loop corrections are suppressed compared to the corresponding CDM+baryons auto-correlation loop corrections. In addition, the suppression is also present to a certain degree at scales larger than the freestreaming scale, due to mode-mode coupling. Moreover, since they are multiplied by $f_{\nu} \ll 1$ (cf. Eq. (4.16)), loop corrections to $P_{\mathrm{cb}, \nu}$ and $P_{\nu, \nu}$ can safely be neglected for an analysis with percent-level accuracy. Nevertheless, we include them for completeness in our analysis.

\subsection{Approximation schemes}

We aim to identify viable simplified approximation schemes to capture the impact of massive neutrinos on loop corrections within the weakly non-linear regime. We therefore compare 
the following schemes, in the order of increasing complexity: ${ }^{2}$

EdS-SPT scheme (1F) The simplest scheme we consider takes neutrinos only into account via their effect on the linear power spectrum,

$$
P_{\mathrm{m}, \mathrm{m}}=P_{\mathrm{m}, \mathrm{m}}^{\text {lin. }}+P_{1 \text {-loop }}^{\text {EdS-SPT }}\left(P_{\mathrm{m}, \mathrm{m}}^{\text {lin. }}\right)+P_{2 \text {-loop }}^{\text {EdST }}\left(P_{\mathrm{m}, \mathrm{m}}^{\text {lin. }}\right),
$$

where $P_{1 \text {-loop }}^{\text {EdST }}\left(P_{\mathrm{m}, \mathrm{m}}\right)$ symbolizes the 1-loop correction computed using EdS-SPT kernels with $P_{\mathrm{m}, \mathrm{m}}$ as input; similarly for 2-loop. While being particularly simple to implement, this scheme is completely agnostic to the scale- and time-dependent free-streaming dynamics of the neutrinos beyond the linear level. In addition, inaccuracies emerge at late times due to the EdS approximation becoming increasingly unreliable (see Sec. 3). We refer to this scheme as $1 \mathrm{~F}$ in the following.

It turns out that the $1 \mathrm{~F}$-scheme underestimates the loop correction to the power spectrum by a factor $\sim 2 L f_{\nu}, L$ being the loop order, for scales much smaller than the freestreaming scale. This will be discussed more below; at this point we note that part of the motivation for the next scheme is to correct for this.

EdS-SPT CDM+baryons scheme (1F-cb) The second scheme is similar to the first one, but uses instead the CDM+baryons linear power spectrum as input for the EdS-SPT loop corrections $[37,59]$ :

$$
P_{\mathrm{m}, \mathrm{m}}=P_{\mathrm{m}, \mathrm{m}}^{\text {lin. }}+\left(1-f_{\nu}\right)^{2} P_{1 \text {-loop }}^{\text {EdS-SPT }}\left(P_{\mathrm{cb}, \mathrm{cb}}^{\text {lin. }}\right)+\left(1-f_{\nu}\right)^{2} P_{2 \text {-loop }}^{\text {EdS-SPT }}\left(P_{\mathrm{cb}, \mathrm{cb}}^{\text {lin. }}\right) .
$$

As the $1 \mathrm{~F}$ scheme, this is reasonably straightforward to implement, but it suffers from the same imprecisenesses. It will be labeled $1 \mathrm{~F}$-cb from now on.

External source scheme (1F-ext) The effect on the CDM and baryons due to the presence of massive neutrinos enters through the coupling via gravitation, and one way to approximate this is to use the linear neutrino transfer function in the Poisson equation [60]. In our notation, this scheme (which we label $1 \mathrm{~F}$-ext) amounts to using

$$
\Omega(k, \eta)=\left(\begin{array}{cc}
0 & -1 \\
-\frac{3}{2} \frac{\Omega_{m}}{f^{2}} \xi(k, \eta) & \frac{3}{2} \frac{\Omega_{m}}{f^{2}}-1
\end{array}\right)
$$

in the equations of motion for a one-component fluid $\psi_{a}=\left(\delta_{\mathrm{cb}},-\theta_{\mathrm{cb}} / \mathcal{H} f\right)$ with

$$
\xi(k, \eta)=1-f_{\nu}+f_{\nu}\left(\frac{\delta_{\nu}(k, \eta)}{\delta_{\mathrm{cb}}(k, \eta)}\right)_{\text {lin. }},
$$

and $\left(\delta_{\nu} / \delta_{\mathrm{cb}}\right)_{\text {lin. }}$ taken as external input. This scheme is readily realized in our numerical setup for loop corrections in models with time- and scale-dependent growth. By the same arguments as for the two-fluid model (and to perform a comparison on equal footing), we use the 1F-ext scheme in a hybrid setup: using the Boltzmann hierarchy (CLASS) up until a redshift $z_{\text {match }}$ before non-linearities become important and matching onto the one-component fluid. We use again $z_{\text {match }}=25$, and take the linear transfer function ratio $\left(\delta_{\nu} / \delta_{\mathrm{cb}}\right)_{\text {lin. }}$. from CLASS.

Analogously to the two-fluid schemes, one needs to carefully choose the initial conditions for the kernels in the 1F-ext scheme, in order to avoid transient modes still being present at

\footnotetext{
${ }^{2}$ We do not consider the scheme of Ref. [58], due to broken momentum conservation and consequently spurious behavior in the $k \rightarrow 0$ limit [33].
} 
$\eta=0$. The prescription for initial conditions described in the beginning of this section may equally well be applied in the $1 \mathrm{~F}$-ext model: we evolve the kernel hierarchy from an early time $\eta_{\text {asymp }} \ll \eta_{\text {match }}$ with $\Omega=\Omega\left(k, \eta_{\text {match }}\right)$ to ensure that the kernels are fully settled in the growing mode solution at $\eta_{\text {match }}$.

Two-fluid scheme with adiabatic sound velocity (2F-ad) In this scheme the neutrino density contrast and velocity divergence are included as a separate component, using the adiabatic approximation for the neutrino sound velocity and neglecting the anisotropic stress, as discussed in Sec. 4 and at the beginning of this section.

Two-fluid scheme with exact effective sound velocity (2F) Finally, the $2 \mathrm{~F}$ scheme is the one which best captures the neutrino dynamics and its impact on the CDM+baryons fluid beyond the linear level, hence we use it as the benchmark for the comparison. It incorporates the full scale-dependent sound velocity as well as the impact of anisotropic stress on the linear evolution matrix. The $2 \mathrm{~F}$ scheme was described in detail in Sec. 4, and the generalization to non-linear corrections was laid out in the beginning of this section.

\subsection{Comparison}

In the following, we discuss the performance of the various approximation schemes in capturing the effect of massive neutrinos on the non-linear power spectrum. The results are summarized in Figs. 7 and 8, which show the non-linear corrections in the different schemes normalized to the $2 \mathrm{~F}$ scheme for various neutrino masses at redshift $z=0$ and $z=0.5$, respectively. At large scales, $k \lesssim 0.1 \mathrm{~h} / \mathrm{Mpc}$, the linear power spectrum dominates and all schemes largely agree. The tiny discrepancies at the permille level arise due to the slight difference between the linear two-fluid model and CLASS, as discussed in Sec. 4. This could easily be corrected for by using the linear power spectrum from CLASS also in the $2 \mathrm{~F}$ and $2 \mathrm{~F}$-ad schemes. At smaller scales, the non-linear corrections become important, and the approximations $1 \mathrm{~F}$ and $1 \mathrm{~F}$-cb perform progressively worse for increasing wavenumber. The $1 \mathrm{~F}$-ext and 2F-ad schemes on the other hand capture the time- and scale-dependence of the dynamics more thoroughly, and remain in good agreement with $2 \mathrm{~F}$. A similar behavior is seen at redshift $z=0.5$, although to a lesser degree, since the non-linear corrections are smaller compared to the linear power spectrum at earlier times. We consider each scheme in detail in what follows.

EdS-SPT scheme (1F) The separate 1- and 2-loop corrections to the matter power spectrum in the $1 \mathrm{~F}$ scheme is plotted in Fig. 9. Even though small scales are beyond the scope of perturbation theory, we note that the asymptotic behavior for large $k$ can be understood in the following way: in this limit the neutrino contribution to the total power spectrum becomes negligible so that $P_{\mathrm{m}, \mathrm{m}} \simeq\left(1-f_{\nu}\right)^{2} P_{\mathrm{cb}, \mathrm{cb}}$. In the $1 \mathrm{~F}$ scheme, the loop corrections to the CDM+baryons power spectrum is then computed with $\left(1-f_{\nu}\right)^{2} P_{\mathrm{cb}, \mathrm{cb}}$ as initial condition rather than $P_{\mathrm{cb}, \mathrm{cb}}$, leading to a suppression by a factor of $2 L f_{\nu}$ compared to the $2 \mathrm{~F}$ result on these scales, with $L$ being the loop order. This is evident for the 1-loop term at $k \gtrsim 1 h / \mathrm{Mpc}$ in Fig. 9. Due to the coupling of Fourier modes, the suppression is to greater extent present across scales in the 2-loop correction. For the highest neutrino masses, both the 1- and 2-loop corrections are smaller than the corresponding terms in the $2 \mathrm{~F}$ scheme by about $5 \%$ in the mildly non-linear regime. Since the 1- and 2-loop terms are of the same order in this domain, but with opposite sign, the deviations largely cancel out, leaving a remarkable agreement for the total power spectrum, which is apparent in the lower plots of Figs. 7 and 8. Due to 

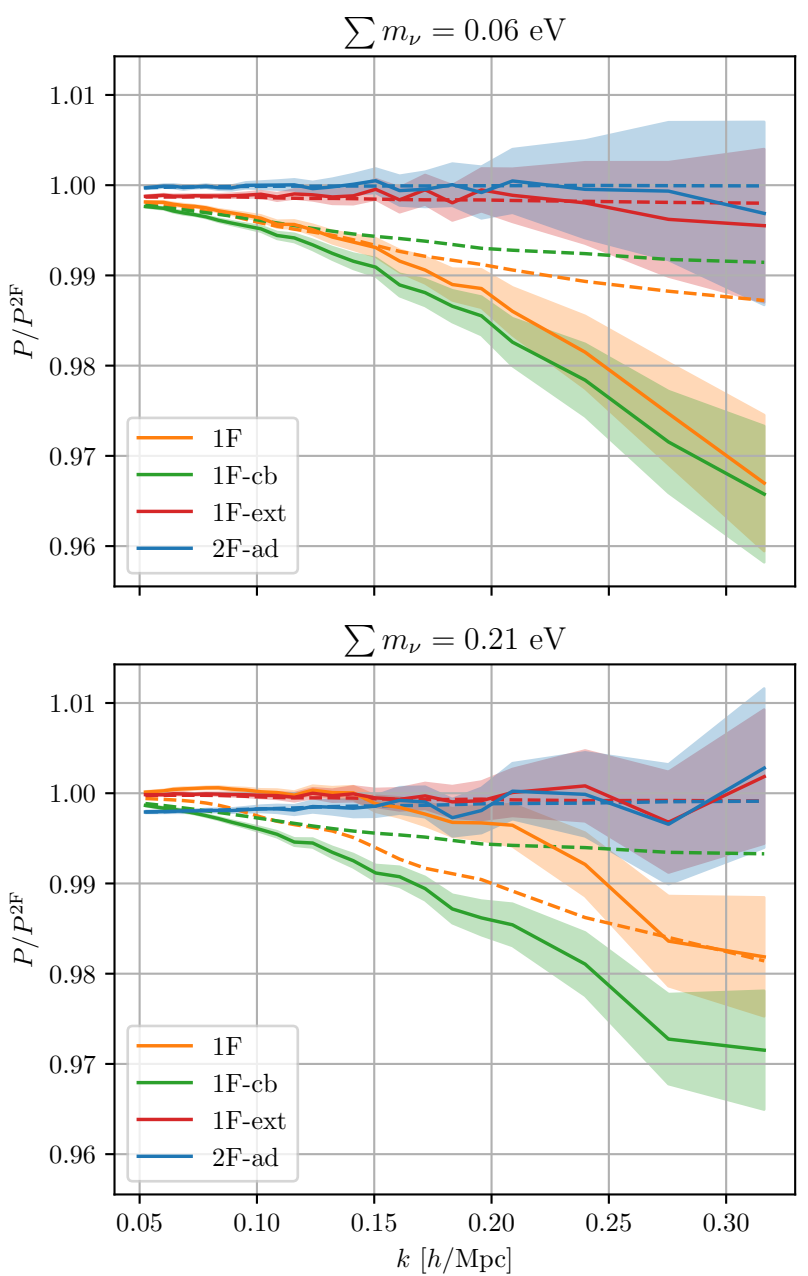
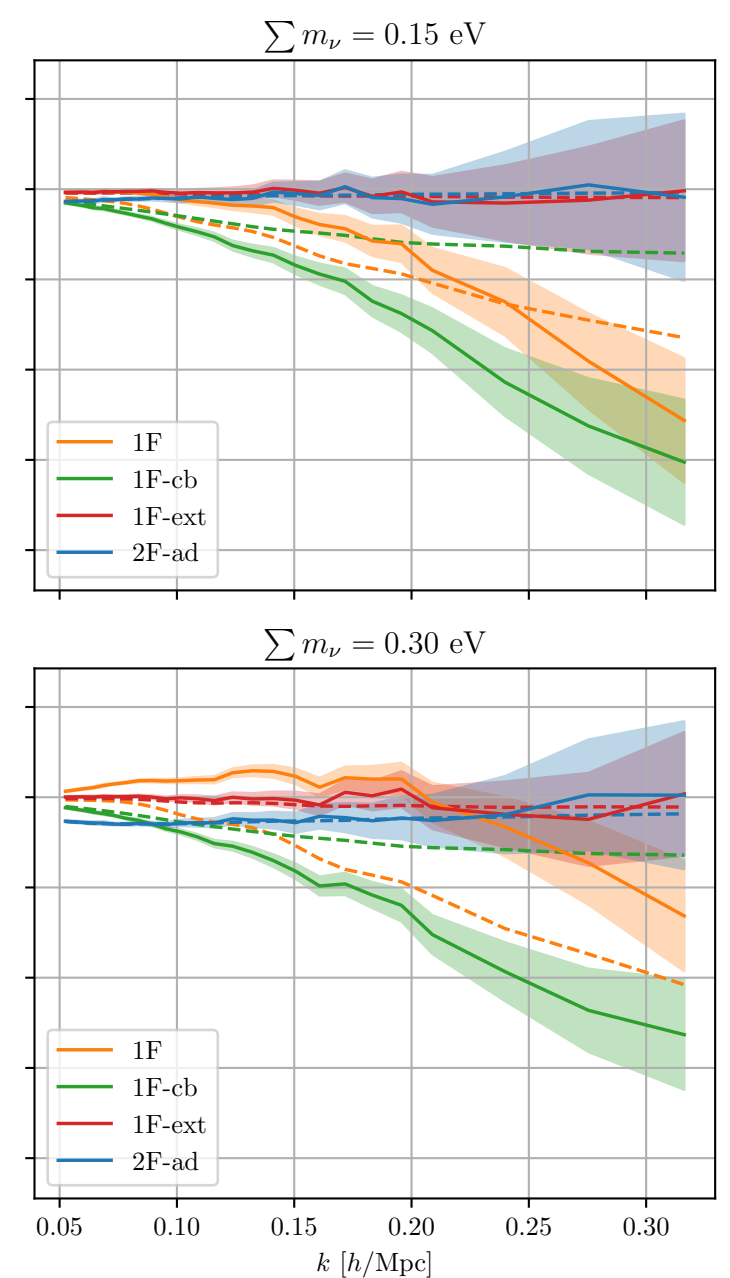

Figure 7. Fractional difference between different schemes and the $2 \mathrm{~F}$ scheme of the total matter power spectrum $P_{\mathrm{m}, \mathrm{m}}$ at redshift $z=0$ and with $\sum m_{\nu}=0.06,0.15,0.21,0.3 \mathrm{eV}$. Dashed lines correspond to the 1-loop power spectrum $P=P^{\text {lin. }}+P^{\text {1-loop}, ~ w h i l e ~ s o l i d ~ l i n e s ~ c o r r e s p o n d ~ t o ~ t h e ~ 2-l o o p ~ p o w e r ~}$ spectrum $P=P^{\text {lin. }}+P^{\text {1-loop }}+P^{2 \text {-loop }}$. The shaded areas indicate the numerical uncertainties of the 2-loop results (numerical uncertainty at 1-loop is invisible). Note that the apparent agreement of the $1 \mathrm{~F}$ and $2 \mathrm{~F}$ schemes at 2-loop for $\sum m_{\nu}=0.15,0.21,0.3 \mathrm{eV}$ and small $k$ is due to an accidental cancellation of inaccuracies in the individual 1- and 2-loop contributions that have opposite sign (see text for details and Fig. 9). Therefore, the total power spectrum cannot be regarded as a faithful measure of the precision of the $1 \mathrm{~F}$ scheme (i.e. it performs worse than one would conclude from a comparison of the power spectrum only), in contrast to $1 \mathrm{~F}$-cb.

the different dependence of the 1- and 2-loop corrections on redshift and other cosmological parameters such as $A_{s}$, this cancellation should be considered as coincidental.

EdS-SPT CDM+baryons scheme (1F-cb) By taking the CDM+baryons power spectrum as input to the EdS-SPT loop evaluation, the $1 \mathrm{~F}$-cb scheme corrects for the $\sim 2 L f_{\nu}$ suppression in the $1 \mathrm{~F}$ scheme. This is seen in Fig. 10, which displays the separate 1- and 2-loop corrections to the power spectrum in the $1 \mathrm{~F}$-cb scheme, normalized to the $2 \mathrm{~F}$ scheme. Given our knowledge of the deviation in the non-linear corrections due to the departure from 

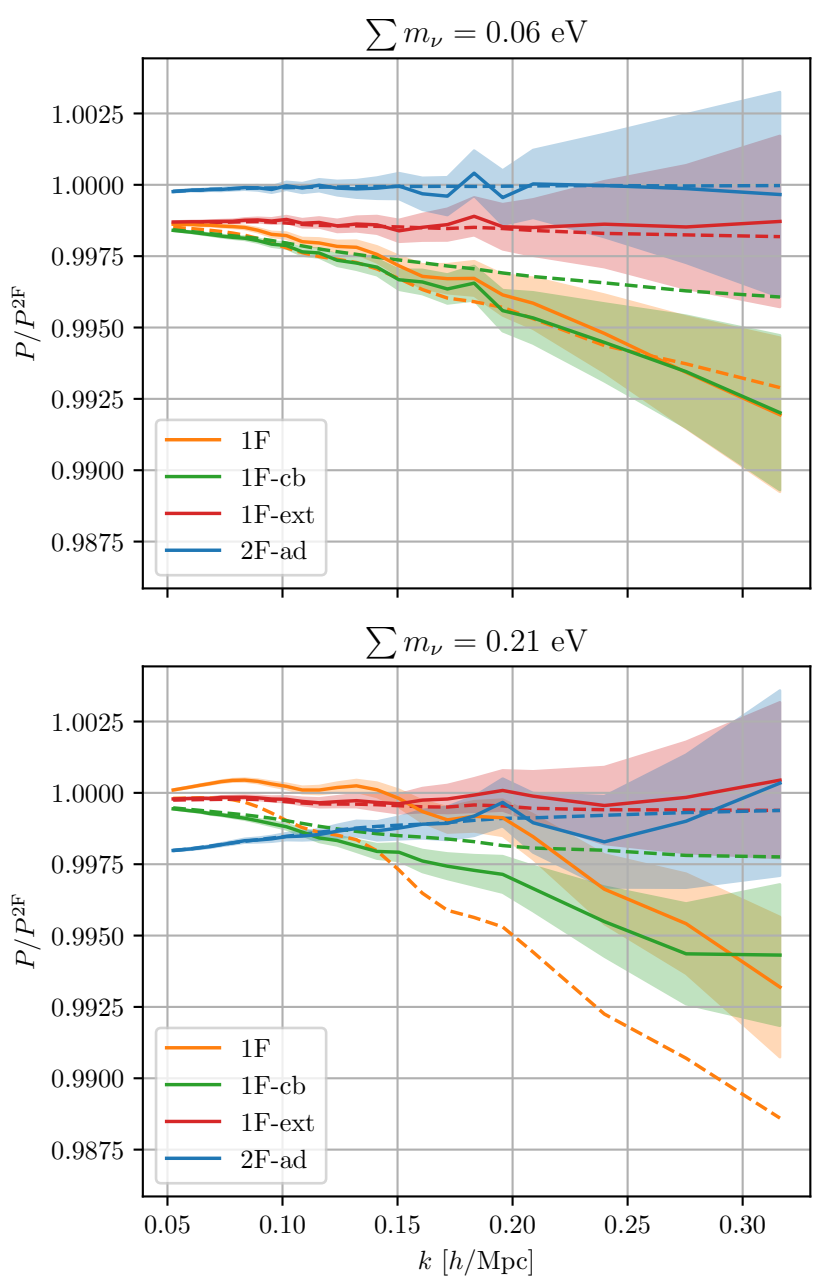
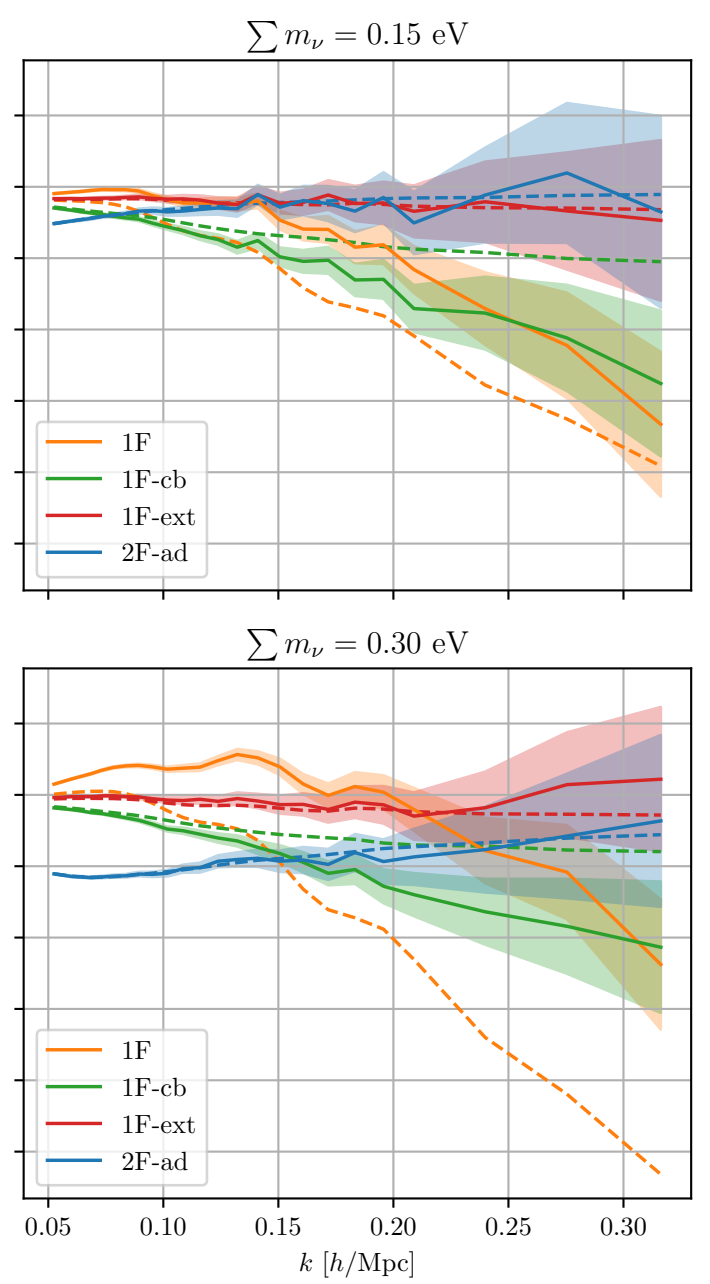

Figure 8. Same as Fig. 7, at redshift $z=0.5$.

EdS from Sec. 3 and comparing to Fig. 1 in particular, we conclude that the main difference between the $1 \mathrm{~F}$-cb and $2 \mathrm{~F}$ results comes from the EdS approximation in $1 \mathrm{~F}$-cb. The deviation due to the neglection of the neutrino-neutrino and CDM+baryons-neutrino power spectra is only about $0.1 \%$. Note that the $1 \mathrm{~F}$-cb scheme appears to perform worse than the $1 \mathrm{~F}$ scheme in Figs. 7 and 8, however this is only due to the accidental cancellation between inaccuracies of the 1- and 2-loop terms in the $1 \mathrm{~F}$ scheme.

External source scheme (1F-ext) With a more proper treatment of the time- and scaledependence of the dynamics, the external source scheme yields results that are unwaveringly in agreement with the $2 \mathrm{~F}$ scheme. There is a small difference at the permille level for the smallest neutrino mass, but this could equally well be attributed to the two-fluid model: it differs about a permille to the CLASS solution at the linear level, and this error plausibly propagates to higher orders. We note that spurious dipole contributions may appear in the $k \rightarrow 0$ limit of the power spectrum in the $1 \mathrm{~F}$-ext scheme, due to momentum conservation being slightly broken [33]. Moreover, the numerical complexity of the $1 \mathrm{~F}$-ext scheme is comparable to the two-fluid setup, such that in practice there is little advantage over using 
$1 \mathrm{~F}, z=0$
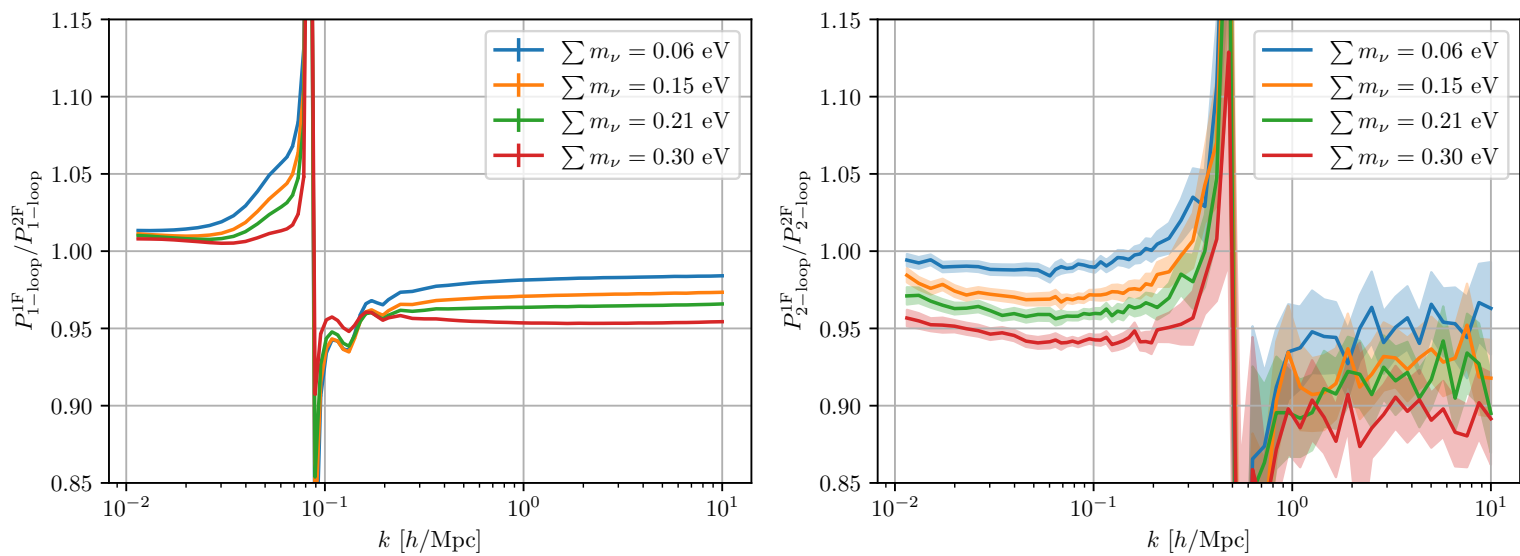

Figure 9. Non-linear corrections to the power spectrum for various neutrino masses at $z=0$ computed in the $1 \mathrm{~F}$ scheme, normalized to the $2 \mathrm{~F}$ result. Left: 1-loop correction. Right: 2-loop correction. Shaded regions indicate uncertainty from numerical integration. At $k \simeq 0.08 h / \mathrm{Mpc}$ and $k \simeq 0.5 h / \mathrm{Mpc}$ the 1 - and 2-loop corrections cross zero, respectively, leading to large relative deviations.

$1 \mathrm{~F}-\mathrm{cb}, z=0$
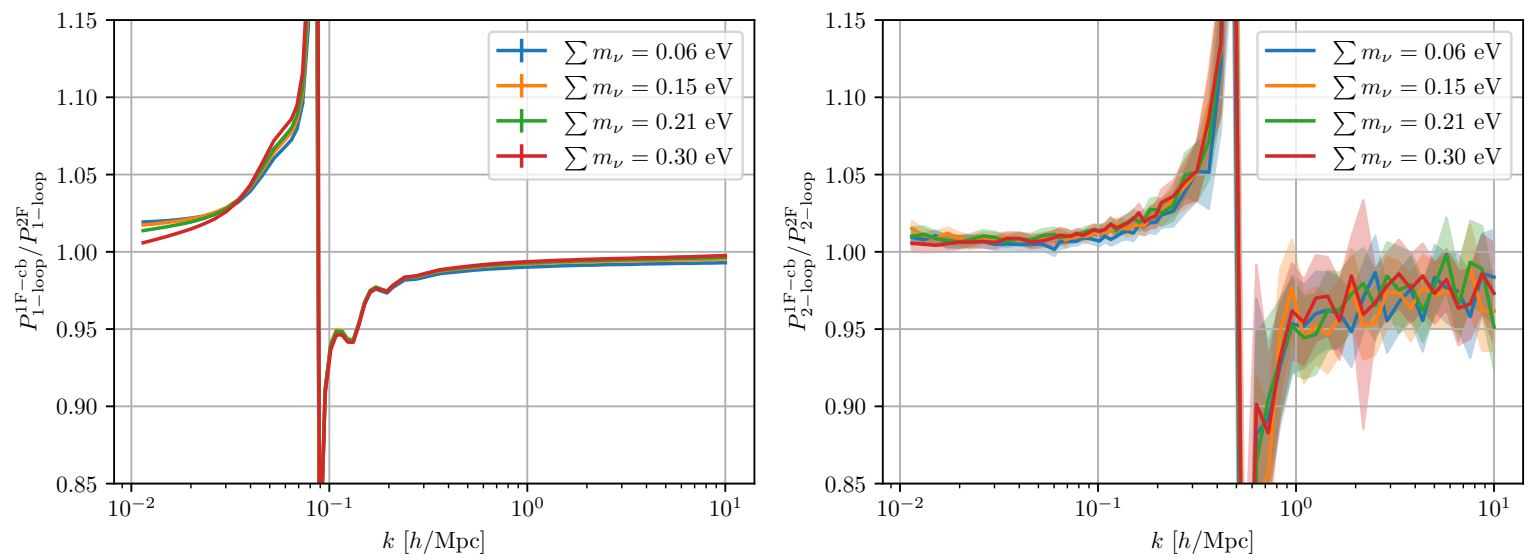

Figure 10. Same as Fig. 9 with the $1 \mathrm{~F}$-cb scheme normalized to $2 \mathrm{~F}$.

$2 \mathrm{~F}$ or $2 \mathrm{~F}$-ad.

Two-fluid scheme with adiabatic sound velocity (2F-ad) Lastly, the $2 \mathrm{~F}$-ad scheme also agrees reasonably well with the $2 \mathrm{~F}$ scheme in the linear and mildly non-linear regimes. Even though the neutrino transfer functions are underestimated by $\mathcal{O}(10 \%)$ at the linear level, this has negligible impact on the total matter power spectrum for the lowest neutrino mass. Increasing the neutrino mass, the error in the neutrino sector influences the CDM+baryons to greater extent via the coupling through gravity, leading to e.g. $0.3 \%$ deviation in the mildly non-linear regime for $\sum m_{\nu}=0.3 \mathrm{eV}$ compared to the $2 \mathrm{~F}$ scheme.

In summary, we find that the external source scheme $1 \mathrm{~F}$-ext best emulates the results of the $2 \mathrm{~F}$ scheme. Of the simplest schemes $1 \mathrm{~F}$ and $1 \mathrm{~F}-\mathrm{cb}, 1 \mathrm{~F}-\mathrm{cb}$ yields the best results for the separate loop corrections, with the main source of error coming from the EdS approximation. 
Even though the $1 \mathrm{~F}$ scheme appears to perform better when the linear and non-linear contributions are added, this is due to a cancellation of errors, which is regarded as coincidental and moreover not expected to necessarily occur when e.g. including redshift space distortion effects or effective field theory corrections.

\section{Conclusions}

In this work, we have set up a framework that captures the effect of time- and scale-dependent growth on non-linear corrections to the power spectrum. This is achieved by extending SPT to a generic form that describes a multi-component fluid with the linear evolution characterized by a time- and scale-dependent matrix $\Omega(k, \eta)$. The framework can be applied to a wide range of extended cosmological models, and we use it in particular to examine the reliability of the EdS approximation and the effect of massive neutrinos on loop corrections to the matter power spectrum. As in SPT, we expand the density contrast and velocity divergence in terms of the linear density contrast, but due to the dependence on time and scale, the resulting equations of motion for the kernels cannot be solved analytically as is possible in the EdS-SPT case. Instead we solve the hierarchical differential equations for the kernels (Eq. (2.13)) numerically, for each configuration of wavenumbers needed by the Monte-Carlo loop integration that yields the non-linear corrections to the power spectrum in the perturbative expansion.

Our first application of the numerical framework is investigating the validity of the commonly used EdS approximation for the matter power spectrum at 2-loop. We checked that at 1-loop, we reproduce the results previously derived in the literature: the EdS approximation is accurate to less than a percent at redshift $z=0$ in the mildly non-linear regime. The deviation increases to more than $1 \%$ at $k=0.2 \mathrm{~h} / \mathrm{Mpc}$ when adding the 2-loop term. At redshift $z=0.5$, the error introduced by the EdS approximation is less, because $\Omega_{m} / f^{2}$ is closer to unity and the non-linear corrections are suppressed to greater extent compared to the linear power spectrum.

In order to be able to calculate non-linear corrections to the power spectrum in the presence of massive neutrinos, we employ a refined version of the two-fluid model of Ref. [33]. It utilizes the fact that after the non-relativistic transition but before non-linearities become important, the lower and higher moments of the Boltzmann hierarchy decouple, so the neutrinos may be described by a fluid with an effective sound velocity. Thereby the CDM+baryons and neutrinos are modeled as separate components of a two-component fluid, with coupling through gravity. We demonstrate two ways to model the effective neutrino sound velocity: using an adiabatic approximation and integrating the linearized Boltzmann equation to obtain sound velocity and anisotropic stress. Comparing the latter with the Boltzmann solver CLASS, we obtain permille and percent agreement for the CDM+baryons and neutrinos, respectively.

The two-fluid setup for massive neutrino cosmologies is readily realized in our numerical framework for investigating the impact of neutrino free-streaming on weakly non-linear scales. We compare the 1- and 2-loop matter power spectrum with full time- and scale-dependence to various simplified approximations that treat the neutrinos linearly. The approximation scheme that best captures the neutrino backreaction on the CDM+baryons is the external source scheme (1F-ext), which we find to agree excellently with the two-fluid model. However, the computational complexity for $1 \mathrm{~F}$-ext is comparable to the full two-fluid treatment. Approximating the loop corrections by $P^{\text {EdS-SPT }}\left(P_{\mathrm{m}, \mathrm{m}}^{\text {lin. }}\right)(1 \mathrm{~F}$ scheme), we find deviations of 
several percent for the individual 1- and 2-loop corrections compared to the two-fluid model. While the mismatch largely cancels when adding 1- and 2-loop contributions, this cancellation among terms of different order in the perturbative expansion should be considered as purely accidental. When using only the $\mathrm{CDM}+$ baryon power spectrum as input to compute loop corrections, $P^{\text {EdS-SPT }}\left(P_{\mathrm{cb}, \mathrm{cb}}^{\text {lin. }}\right)$ (1F-cb scheme), the 1- and 2-loop corrections are in better agreement with the two-fluid treatment. The total matter power spectrum deviates by about $1 \%(z=0)$ and $0.25 \%(z=0.5)$ from the two-fluid model at $k=0.15 \mathrm{~h} / \mathrm{Mpc}$, with the predominant error coming from the EdS approximation.

Given the versatility of our numerical framework, it is suitable for precision analyses incorporating relativistic effects, small-scale effective stress-tensor corrections and redshift space distortions in addition to describing extended cosmological models in the future.

\section{Acknowledgments}

This work was supported by the DFG Collaborative Research Institution Neutrinos and Dark Matter in Astro- and Particle Physics (SFB 1258).

\section{A Validation with analytic 1-loop kernels}

In this appendix, we compare the power spectrum at 1-loop obtained using numerically evolved kernels and using analytic expressions for the kernels when the EdS approximation is relaxed. Expressions for the second- and third-order generalized kernels with timedependence have been derived in the past [48-52], and we repeat them here for convenience. At second order, the kernels may be written in such a way that the time-dependence is contained in the angle-averaged kernels $\nu_{2}$ and $\mu_{2}$,

$$
\begin{aligned}
& F_{2}\left(\mathbf{q}_{1}, \mathbf{q}_{2} ; \eta\right)=\left(-\frac{1}{2}+\frac{3}{4} \nu_{2}(\eta)\right) \alpha^{\mathrm{s}}\left(\mathbf{q}_{1}, \mathbf{q}_{2}\right)+\left(\frac{3}{2}-\frac{3}{4} \nu_{2}(\eta)\right) \beta\left(\mathbf{q}_{1}, \mathbf{q}_{2}\right), \\
& G_{2}\left(\mathbf{q}_{1}, \mathbf{q}_{2} ; \eta\right)=\left(-\frac{1}{2}+\frac{3}{4} \mu_{2}(\eta)\right) \alpha^{\mathrm{s}}\left(\mathbf{q}_{1}, \mathbf{q}_{2}\right)+\left(\frac{3}{2}-\frac{3}{4} \mu_{2}(\eta)\right) \beta\left(\mathbf{q}_{1}, \mathbf{q}_{2}\right),
\end{aligned}
$$

where $\alpha^{\mathrm{s}}\left(\mathbf{q}_{1}, \mathbf{q}_{2}\right)=\left(\alpha\left(\mathbf{q}_{1}, \mathbf{q}_{2}\right)+\alpha\left(\mathbf{q}_{2}, \mathbf{q}_{1}\right)\right) / 2$. Note that an overall factor $\mathrm{e}^{n \eta}$ was extracted from the kernels above compared to those defined in Eq. (2.12): in our notation from Sec. 2.2 we have $F_{1}^{(2)}(\eta)=\mathrm{e}^{2 \eta} F_{2}(\eta)$ and $F_{2}^{(2)}(\eta)=\mathrm{e}^{2 \eta} G_{2}(\eta)$. The angle-averaged kernels are momentum-independent and satisfy the following equations of motion:

$$
\partial_{\eta}\left(\begin{array}{c}
\nu_{n} \\
\mu_{n}
\end{array}\right)_{a}+n\left(\begin{array}{c}
\nu_{n} \\
\mu_{n}
\end{array}\right)_{a}+\Omega_{a b}(\eta)\left(\begin{array}{c}
\nu_{n} \\
\mu_{n}
\end{array}\right)_{b}=\sum_{m=1}^{n-1}\left(\begin{array}{c}
n \\
m
\end{array}\right)\left(\begin{array}{c}
\mu_{m} \nu_{n-m} \\
\frac{1}{3} \mu_{m} \mu_{n-m}
\end{array}\right)_{a},
$$

where $\Omega_{a b}$ was defined in Eq. (2.4). Given the linear growing mode solution $\nu_{1}=\mu_{1}=1$, the equation of motion for $\nu_{2}$ and $\mu_{2}$ may easily be integrated numerically. Due to the factorization of the time- and scale-dependence in Eqs. (A.1) and (A.2), one only needs to do this once given a cosmological model. In contrast, the numerical setup described in Sec. 2.2 necessitates solving a similar set of differential equations separately for each wavenumber configuration required by the Monte-Carlo loop integration. Factorizing the time- and scaledependence of generalized kernels becomes progressively more difficult at higher orders in perturbation theory, however; as far as we are aware there is no derivation of this beyond 

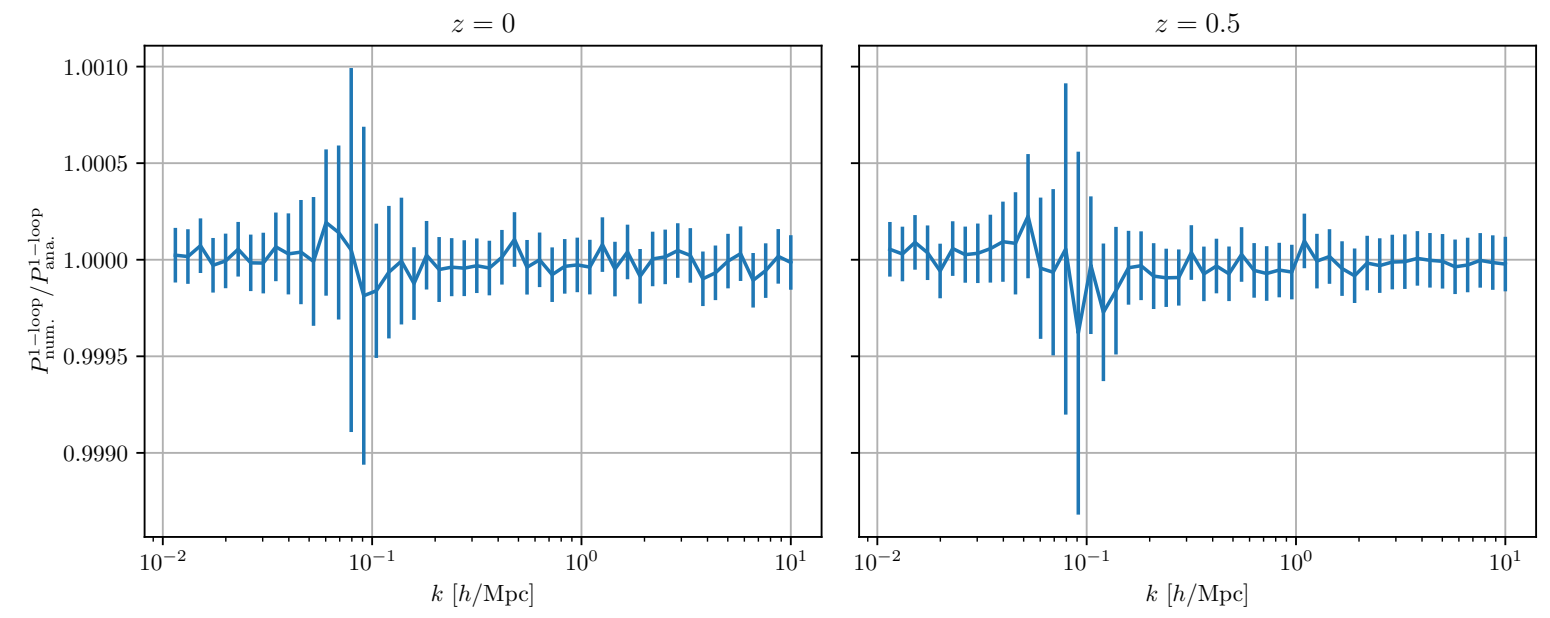

Figure 11. Ratio of the 1-loop correction to the matter power spectrum computed from numerically evolved kernels and computed from the analytic kernel expressions. The $\nu_{2}, \nu_{3}$ and $\lambda_{3}$ parameters used are listed in Table 1. Left: $z=0$. Right: $z=0.5$. The error bars correspond to uncertainty from the numerical integration.

third order. We note finally that in the EdS limit, $\nu_{2}=34 / 21$ and $\mu_{2}=26 / 21$, which inserted in Eqs. (A.1) and (A.2) reproduces the EdS-SPT kernels.

At third order, the time-dependence of the $F_{3}$ kernel can be extracted into the parameters $\nu_{2}, \nu_{3}$ and $\lambda_{3}[16,48],{ }^{3}$

$$
\begin{aligned}
F_{3}\left(\mathbf{q}_{1}, \mathbf{q}_{2}, \mathbf{q}_{3} ; \eta\right)= & \mathcal{R}_{1}\left(\mathbf{q}_{1}, \mathbf{q}_{2}, \mathbf{q}_{3}\right)+\nu_{2}(\eta) \mathcal{R}_{2}\left(\mathbf{q}_{1}, \mathbf{q}_{2}, \mathbf{q}_{3}\right)+\nu_{3}(\eta) \mathcal{R}_{3}\left(\mathbf{q}_{1}, \mathbf{q}_{2}, \mathbf{q}_{3}\right) \\
& +\lambda_{3}(\eta) \mathcal{R}_{4}\left(\mathbf{q}_{1}, \mathbf{q}_{2}, \mathbf{q}_{3}\right),
\end{aligned}
$$

where $\lambda_{3}(\eta)$ is a function satisfying

$$
\left(\partial_{\ln a}^{2}+\left(1+\frac{\mathrm{d} \ln \mathcal{H}}{\mathrm{d} \ln a}\right) \partial_{\ln a}-\frac{3}{2} \Omega_{m}\right)\left(\lambda_{3}(a) D^{3}(a)\right)=\frac{3}{2} \Omega_{m} D^{3}(a) .
$$

Using the notations $\alpha_{i j, k}=\alpha\left(\mathbf{q}_{i}+\mathbf{q}_{j}, \mathbf{q}_{k}\right)$ and $\alpha_{i, j k}=\alpha\left(\mathbf{q}_{i}, \mathbf{q}_{j}+\mathbf{q}_{k}\right)$, the momentumdependent functions in the expression for $F_{3}$ are

$$
\begin{aligned}
& \mathcal{R}_{1}=\left(\frac{2}{3} \alpha_{3,12}^{\mathrm{s}}+\frac{1}{3} \beta_{3,12}\right) \alpha_{1,2}+\left(\frac{1}{6} \alpha_{3,12}-\frac{1}{2} \alpha_{3,12}^{\mathrm{s}}-\frac{5}{2} \beta_{3,12}\right)\left(\alpha_{1,2}^{\mathrm{s}}-\beta_{1,2}\right), \\
& \mathcal{R}_{2}=\frac{3}{4}\left(3 \beta_{3,12}-\alpha_{3,12}^{\mathrm{s}}\right)\left(\alpha_{1,2}^{\mathrm{s}}-\beta_{1,2}\right) \\
& \mathcal{R}_{3}=\frac{3}{8}\left(\alpha_{3,12}^{\mathrm{s}}-\beta_{3,12}\right)\left(\alpha_{1,2}^{\mathrm{s}}-\beta_{1,2}\right) \\
& \mathcal{R}_{4}=\frac{2}{3}\left(\alpha_{3,12}^{\mathrm{s}}-\beta_{3,12}\right) \alpha_{1,2}-\left(\frac{1}{3} \alpha_{3,12}+\frac{1}{2} \alpha_{3,12}^{\mathrm{s}}-\frac{1}{2} \beta_{3,12}\right)\left(\alpha_{1,2}^{\mathrm{s}}-\beta_{1,2}\right) .
\end{aligned}
$$

We symmetrize the above expressions with respect to permutations of $\mathbf{q}_{1}, \mathbf{q}_{2}$ and $\mathbf{q}_{3}$ in order to utilize the loop integration algorithm described in Sec. 2.2.

\footnotetext{
${ }^{3} \mathrm{~A}$ similar expression for $F 3$ is derived in Ref. [51], which is related to Eq. (A.4) by $\lambda_{1}=8 / 9-\nu_{2}+\nu_{3} / 2+$ $2 \lambda_{3} / 9$ and $\lambda_{2}=13 / 18-3 \nu_{2} / 4+3 \nu_{3} / 8+\lambda_{3} / 18$.
} 
Table 1. Parameters used in expressions for generalized kernels. The constant values in EdS are listed as reference.

\begin{tabular}{lllll}
\hline & $\nu_{2}$ & $\mu_{2}$ & $\nu_{3}$ & $\lambda_{3}$ \\
\hline EdS & $34 / 21$ & $26 / 21$ & $682 / 189$ & $1 / 6$ \\
$\Lambda \mathrm{CDM}, z=0$ & 1.6215126 & 1.2510105 & 3.6228998 & 0.1696433 \\
$\Lambda \mathrm{CDM}, z=0.5$ & 1.6201647 & 1.2434840 & 3.6149456 & 0.1679576 \\
\hline
\end{tabular}

In Fig. 11, we compare the power spectrum at 1-loop using kernels computed from Eqs. (A.1), (A.2) and (A.4) with that computed by solving Eq. (2.13) (with $\Omega$ given in Eq. (2.4)) numerically. The time-dependent parameters in the generalized kernels are evaluated numerically by solving Eqs. (A.3) and (A.5), yielding the values listed in Table 1. We find excellent agreement between the power spectra from the analytic kernels and from the numerically evolved ones.

\section{B Numerical checks of convergence}

We performed various numerical checks to ensure the stability of the algorithm. First of all, we checked that when using the EdS-SPT recursion relation for the kernels (rather than evolving them numerically), we obtain agreement between two independent implementations computing the 2-loop power spectrum. Secondly, when evolving the kernels numerically according to Eq. (2.13) using the constant evolution matrix $\Omega=\Omega^{\mathrm{EdS}}$, we confirm that we recover the results of the EdS-SPT recursion relation. Finally, we checked that changing various parameters in the numerical setup does not change the results beyond the numerical uncertainty. We discuss the last point in detail in the following.

As described in Sec. 2.2, for each wavenumber configuration the kernels computed by Eq. (2.13) are temporarily stored on a grid between the initial and final times, so that they can potentially be used in the RHS of the same equation when solving for a higher order kernel. We used $N=100$ as grid size in both the analysis of the departure from EdS in Sec. 3 and the analysis of the effect of massive neutrinos on the matter power spectrum in Sec. 5 . For the one-fluid implementation used in Sec. 3, increasing the grid size to $N=150$ does not alter the results beyond the numerical uncertainty. Likewise, setting $N=125$ in the two-fluid model for massive neutrinos (scheme 2F) impacts the results insignificantly. In Fig. 12 we show the relative difference between results obtained with $N=125$ and $N=100$ for a subset of wavenumbers and for all the 1- and 2-loop corrections to the total power spectrum in a cosmology with neutrino mass $\sum m_{\nu}=0.15 \mathrm{eV}$. At small scales, there is a relative deviation of order $\mathcal{O}(1)$ in $P_{\mathrm{cb}, \nu}$ and $P_{\nu, \nu}$. However, $P_{\mathrm{cb}, \nu}$ and $P_{\nu, \nu}$ are suppressed by several orders of magnitude compared to $P_{\mathrm{cb}, \mathrm{cb}}$ in that domain, and the total power spectrum receives no significant change from altering $N$. In addition, small scales are anyway beyond the reach of perturbation theory; in the linear and mildly non-linear regime none of the contributions differ beyond the numerical uncertainties when changing $N$. We perform the same check for the other neutrino masses considered in this work, also finding deviations that are smaller than the numerical uncertainty.

We used throughout $q_{\min }=10^{-4} h / \mathrm{Mpc}$ and $q_{\max }=65 \mathrm{~h} / \mathrm{Mpc}$ as integration limits in the Monte-Carlo integration. This domain covers the scales in which the linear power 

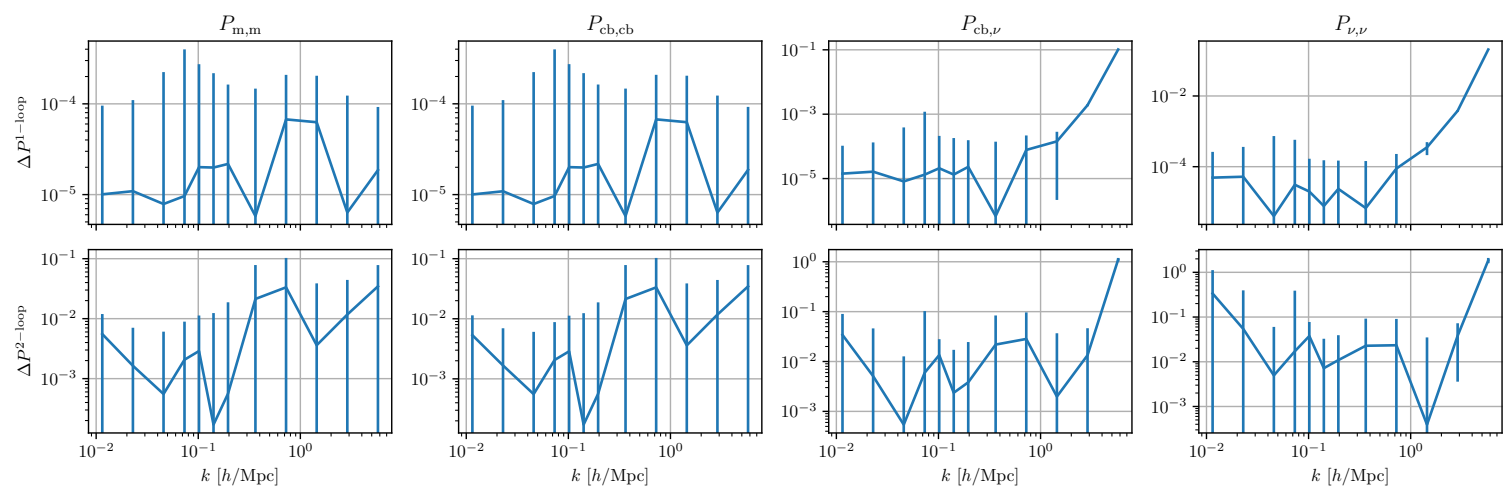

Figure 12. Relative difference of the various contributions to the 1- and 2-loop corrections to the power spectrum when changing the number of grid points between the initial and final times, i.e. $\Delta P=\left|P^{N=125} / P^{N=100}-1\right|$. Neutrino mass: $\sum m_{\nu}=0.15 \mathrm{eV}$. The top and bottom rows correspond to the 1- and 2-loop correction, respectively. The columns display from left to right: $P_{\mathrm{m}, \mathrm{m}}, P_{\mathrm{cb}, \mathrm{cb}}, P_{\mathrm{cb}, \nu}$ and $P_{\nu, \nu}$.
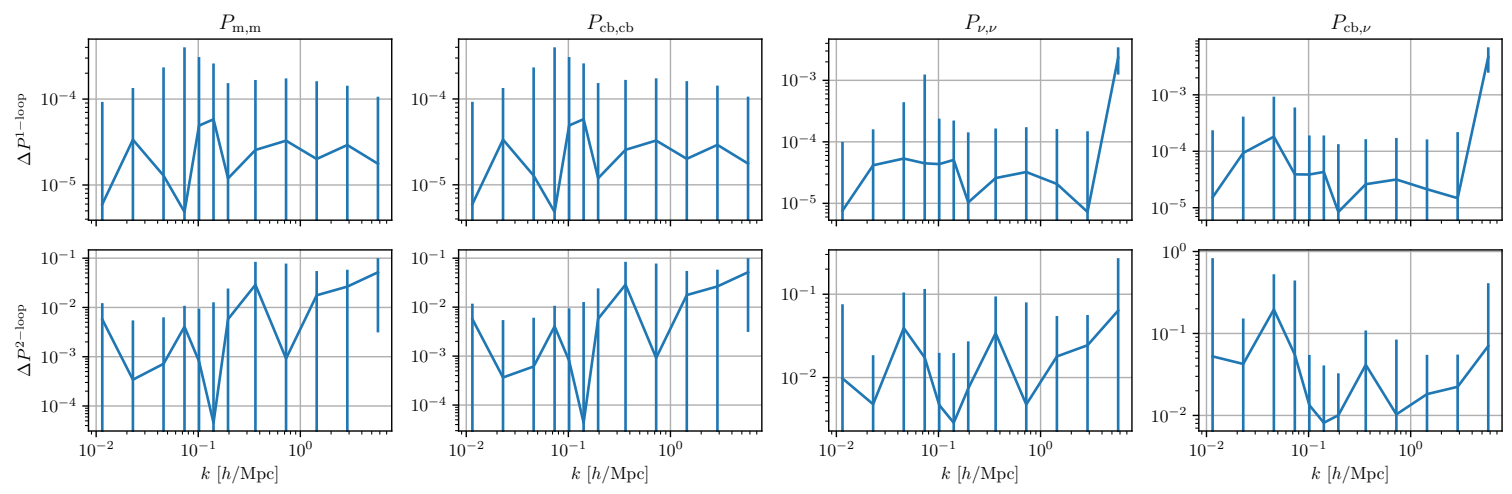

Figure 13. Relative difference of the various contributions to the 1- and 2-loop corrections to the power spectrum when adjusting the lower integration cutoff in the loop integration, i.e. $\Delta P=$ $\left|P\left(q_{\min }=1.3 \cdot 10^{-5} h / \mathrm{Mpc}\right) / P\left(q_{\min }=10^{-4} h / \mathrm{Mpc}\right)-1\right|$. Neutrino mass: $\sum m_{\nu}=0.15 \mathrm{eV}$. The top and bottom rows correspond to the 1- and 2-loop correction, respectively. The columns display from left to right: $P_{\mathrm{m}, \mathrm{m}}, P_{\mathrm{cb}, \mathrm{cb}}, P_{\mathrm{cb}, \nu}$ and $P_{\nu, \nu}$.

spectrum is largest and hence also the regions where the integrand receives its dominant contributions. Indeed, beyond the chosen integration limits the linear power spectrum is suppressed by several orders of magnitude compared to the peak. Moreover, due to the cancellations of large terms in the integrand when the external wavenumber is large, one cannot extend the upper limit too far: the terms in question grow with increasing separation between the momenta (both loop and external) and at some point the cancellation might be slightly spoiled by machine rounding error. We find that using an upper integration limit around $q_{\max }=65$, the one-fluid calculation used in Sec. 3 is stable against modest changes of $q_{\text {max }}$. In addition, decreasing the lower integration limit to $q_{\min }=1.3 \cdot 10^{-5}$ does not alter the results beyond the numerical error bars. A similar exercise was performed for the two-fluid model with massive neutrinos: in Fig. 13 we show the relative difference introduced in the 1- and 2-loop corrections when decreasing the lower integration limit to $1.3 \cdot 10^{-5}$ (neutrino 

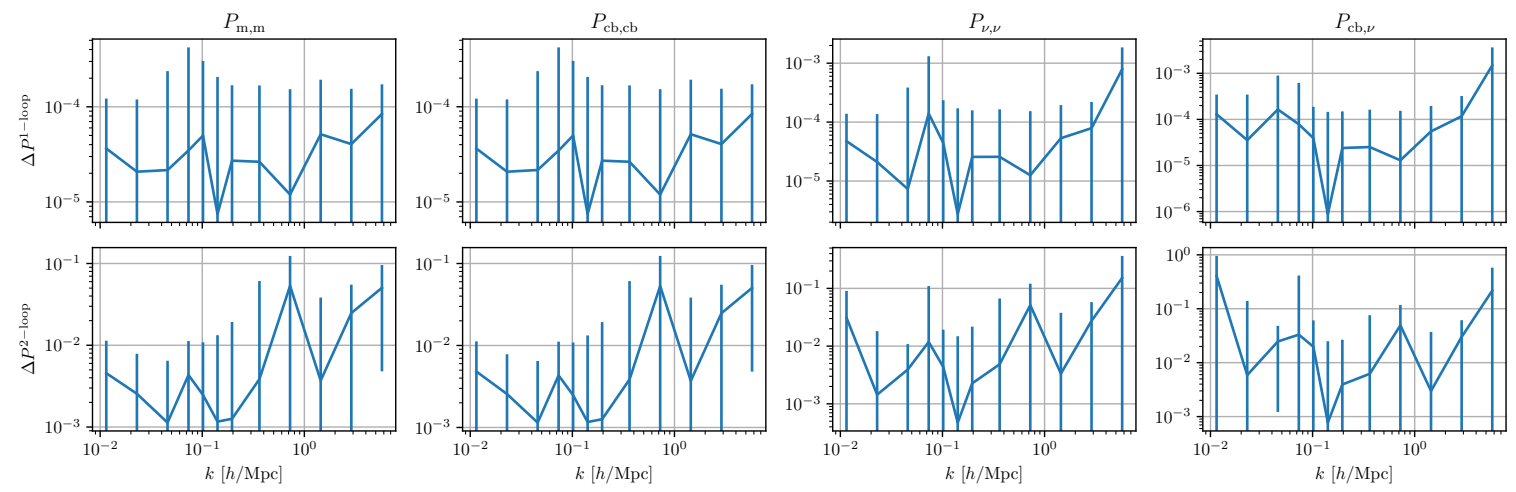

Figure 14. Relative difference of the various contributions to the 1- and 2-loop corrections to the power spectrum when adjusting the upper integration cutoff in the loop integration, i.e. $\Delta P=$ $\left|P\left(q_{\max }=70 h / \mathrm{Mpc}\right) / P\left(q_{\max }=65 h / \mathrm{Mpc}\right)-1\right|$. Neutrino mass: $\sum m_{\nu}=0.15 \mathrm{eV}$. The top and bottom rows correspond to the 1- and 2-loop correction, respectively. The columns display from left to right: $P_{\mathrm{m}, \mathrm{m}}, P_{\mathrm{cb}, \mathrm{cb}}, P_{\mathrm{cb}, \nu}$ and $P_{\nu, \nu}$.

mass $\left.\sum m_{\nu}=0.15 \mathrm{eV}\right)$. Corresponding plots for the relative difference between results with $q_{\max }=65$ and $q_{\max }=70$ are shown in Fig. 14. We find agreement within the numerical uncertainty both when changing the lower and upper integration cutoffs, except for a few of the values on small scales, which are anyway far beyond the scope of perturbation theory. Similar agreement is found for the other neutrino masses used in this work.

Let us finally comment on the computational complexity of the schemes considered in this work. The main additional running time compared to EdS-SPT kernels is required for solving the ordinary differential equations for the time-dependent kernels. For the fiducial choice of parameters discussed above, the $1 \mathrm{~F}$-ext scheme has an overhead of around a factor 100 compared to $1 \mathrm{~F}$, and $2 \mathrm{~F}$ as well as $2 \mathrm{~F}$-ad of $\sim 500$. For $2 \mathrm{~F}$, the exact sound velocity and anisotropic stress need to be computed in addition. However, they can be pre-computed for a sufficiently dense grid in wavenumber. The running time of $1 \mathrm{~F}$ and $1 \mathrm{~F}$-cb is identical, since both schemes are based on EdS-SPT kernels. In this work we focus on achieving maximal precision, in order to quantify the accuracy of the computationally more efficient schemes $1 \mathrm{~F}$ and $1 \mathrm{~F}$-cb. Nevertheless, by optimizing the numerical settings used in the $1 \mathrm{~F}$-ext and $2 \mathrm{~F} / 2 \mathrm{~F}$-ad schemes, we expect that their running time can be reduced.

\section{References}

[1] DES collaboration, Dark Energy Survey Year 1 Results: Constraints on Extended Cosmological Models from Galaxy Clustering and Weak Lensing, Phys. Rev. D 99 (2019) 123505 [1810.02499].

[2] EBOSS collaboration, Completed SDSS-IV extended Baryon Oscillation Spectroscopic Survey: Cosmological implications from two decades of spectroscopic surveys at the Apache Point Observatory, Phys. Rev. D 103 (2021) 083533 [2007.08991].

[3] SPT collaboration, Cluster Cosmology Constraints from the $2500 \mathrm{deg}^{2}$ SPT-SZ Survey: Inclusion of Weak Gravitational Lensing Data from Magellan and the Hubble Space Telescope, Astrophys. J. 878 (2019) 55 [1812.01679].

[4] L. Amendola et al., Cosmology and fundamental physics with the Euclid satellite, Living Rev. Rel. 21 (2018) 2 [1606.00180]. 
[5] DESI collaboration, The Dark Energy Spectroscopic Instrument (DESI), 1907.10688.

[6] PFS TeAm collaboration, Extragalactic science, cosmology, and Galactic archaeology with the Subaru Prime Focus Spectrograph, Publ. Astron. Soc. Jap. 66 (2014) R1 [1206.0737].

[7] LSST Science, LSST Project collaboration, LSST Science Book, Version 2.0, 0912.0201.

[8] K. Bechtol et al., Dark Matter Science in the Era of LSST, 1903.04425.

[9] V. Gluscevic et al., Cosmological Probes of Dark Matter Interactions: The Next Decade, 1903.05140.

[10] B. Audren, J. Lesgourgues, S. Bird, M. G. Haehnelt and M. Viel, Neutrino masses and cosmological parameters from a Euclid-like survey: Markov Chain Monte Carlo forecasts including theoretical errors, JCAP 01 (2013) 026 [1210.2194].

[11] A. Boyle and E. Komatsu, Deconstructing the neutrino mass constraint from galaxy redshift surveys, JCAP 03 (2018) 035 [1712.01857].

[12] T. Brinckmann, D. C. Hooper, M. Archidiacono, J. Lesgourgues and T. Sprenger, The promising future of a robust cosmological neutrino mass measurement, JCAP 01 (2019) 059 [1808.05955].

[13] S. Mishra-Sharma, D. Alonso and J. Dunkley, Neutrino masses and beyond- $\Lambda$ CDM cosmology with LSST and future CMB experiments, Phys. Rev. D 97 (2018) 123544 [1803.07561].

[14] A. Chudaykin and M. M. Ivanov, Measuring neutrino masses with large-scale structure: Euclid forecast with controlled theoretical error, JCAP 11 (2019) 034 [1907.06666].

[15] W. L. Xu, N. DePorzio, J. B. Muñoz and C. Dvorkin, Accurately Weighing Neutrinos with Cosmological Surveys, Phys. Rev. D 103 (2021) 023503 [2006.09395].

[16] F. Bernardeau, S. Colombi, E. Gaztanaga and R. Scoccimarro, Large scale structure of the universe and cosmological perturbation theory, Phys. Rept. 367 (2002) 1 [astro-ph/0112551].

[17] V. Desjacques, D. Jeong and F. Schmidt, Large-Scale Galaxy Bias, Phys. Rept. 733 (2018) 1 [1611.09787].

[18] R. Scoccimarro, H. Couchman and J. A. Frieman, The Bispectrum as a Signature of Gravitational Instability in Redshift-Space, Astrophys. J. 517 (1999) 531 [astro-ph/9808305].

[19] S. Pueblas and R. Scoccimarro, Generation of Vorticity and Velocity Dispersion by Orbit Crossing, Phys. Rev. D 80 (2009) 043504 [0809.4606].

[20] D. Baumann, A. Nicolis, L. Senatore and M. Zaldarriaga, Cosmological Non-Linearities as an Effective Fluid, JCAP 07 (2012) 051 [1004.2488].

[21] M. Crocce and R. Scoccimarro, Renormalized cosmological perturbation theory, Phys. Rev. D 73 (2006) 063519 [astro-ph/0509418].

[22] L. Senatore and M. Zaldarriaga, The IR-resummed Effective Field Theory of Large Scale Structures, JCAP 02 (2015) 013 [1404.5954].

[23] T. Baldauf, M. Mirbabayi, M. Simonović and M. Zaldarriaga, Equivalence Principle and the Baryon Acoustic Peak, Phys. Rev. D 92 (2015) 043514 [1504.04366].

[24] D. Blas, M. Garny, M. M. Ivanov and S. Sibiryakov, Time-Sliced Perturbation Theory II: Baryon Acoustic Oscillations and Infrared Resummation, JCAP 07 (2016) 028 [1605.02149].

[25] BOSS collaboration, The clustering of galaxies in the completed SDSS-III Baryon Oscillation Spectroscopic Survey: cosmological implications of the configuration-space clustering wedges, Mon. Not. Roy. Astron. Soc. 464 (2017) 1640 [1607.03147].

[26] G. D'Amico, J. Gleyzes, N. Kokron, D. Markovic, L. Senatore, P. Zhang et al., The Cosmological Analysis of the SDSS/BOSS data from the Effective Field Theory of Large-Scale Structure, JCAP 05 (2020) 005 [1909.05271]. 
[27] M. M. Ivanov, M. Simonović and M. Zaldarriaga, Cosmological Parameters from the BOSS Galaxy Power Spectrum, JCAP 05 (2020) 042 [1909.05277].

[28] T. Tröster et al., Cosmology from large-scale structure: Constraining $\Lambda$ CDM with BOSS, Astron. Astrophys. 633 (2020) L10 [1909.11006].

[29] J. Lesgourgues and S. Pastor, Massive neutrinos and cosmology, Phys. Rept. 429 (2006) 307 [astro-ph/0603494].

[30] D. Blas, J. Lesgourgues and T. Tram, The Cosmic Linear Anisotropy Solving System (CLASS) II: Approximation schemes, JCAP 07 (2011) 034 [1104.2933].

[31] C. Howlett, A. Lewis, A. Hall and A. Challinor, CMB power spectrum parameter degeneracies in the era of precision cosmology, JCAP 04 (2012) 027 [1201.3654].

[32] F. Führer and Y. Y. Y. Wong, Higher-order massive neutrino perturbations in large-scale structure, JCAP 03 (2015) 046 [1412.2764].

[33] D. Blas, M. Garny, T. Konstandin and J. Lesgourgues, Structure formation with massive neutrinos: going beyond linear theory, JCAP 11 (2014) 039 [1408.2995].

[34] T. Tram, J. Brandbyge, J. Dakin and S. Hannestad, Fully relativistic treatment of light neutrinos in N-body simulations, JCAP 03 (2019) 022 [1811.00904].

[35] S. Bird, M. Viel and M. G. Haehnelt, Massive Neutrinos and the Non-linear Matter Power Spectrum, Mon. Not. Roy. Astron. Soc. 420 (2012) 2551 [1109.4416].

[36] J. Dakin, J. Brandbyge, S. Hannestad, T. Haugbølle and T. Tram, $\nu$ CON CEPT: Cosmological neutrino simulations from the non-linear Boltzmann hierarchy, JCAP 02 (2019) 052 [1712.03944].

[37] E. Castorina, C. Carbone, J. Bel, E. Sefusatti and K. Dolag, DEMNUni: The clustering of large-scale structures in the presence of massive neutrinos, JCAP 07 (2015) 043 [1505.07148].

[38] S. Hannestad, A. Upadhye and Y. Y. Y. Wong, Spoon or slide? The non-linear matter power spectrum in the presence of massive neutrinos, JCAP 11 (2020) 062 [2006.04995].

[39] D. Blas, M. Garny and T. Konstandin, On the non-linear scale of cosmological perturbation theory, JCAP 09 (2013) 024 [1304.1546].

[40] D. Blas, M. Garny and T. Konstandin, Cosmological perturbation theory at three-loop order, JCAP 01 (2014) 010 [1309.3308].

[41] D. Blas, S. Floerchinger, M. Garny, N. Tetradis and U. A. Wiedemann, Large scale structure from viscous dark matter, JCAP 11 (2015) 049 [1507.06665].

[42] J. J. M. Carrasco, S. Foreman, D. Green and L. Senatore, The 2-loop matter power spectrum and the IR-safe integrand, JCAP 07 (2014) 056 [1304.4946].

[43] T. Hahn, CUBA: A Library for multidimensional numerical integration, Comput. Phys. Commun. 168 (2005) 78 [hep-ph/0404043].

[44] M. Pietroni, Flowing with Time: a New Approach to Nonlinear Cosmological Perturbations, JCAP 10 (2008) 036 [0806.0971].

[45] B. Audren and J. Lesgourgues, Non-linear matter power spectrum from Time Renormalisation Group: efficient computation and comparison with one-loop, JCAP 10 (2011) 037 [1106.2607].

[46] G. Juergens and M. Bartelmann, Perturbation Theory Trispectrum in the Time Renormalisation Approach, 1204.6524.

[47] S. Floerchinger, M. Garny, A. Katsis, N. Tetradis and U. A. Wiedemann, The dark matter bispectrum from effective viscosity and one-particle irreducible vertices, JCAP 09 (2019) 047 [1907.10729]. 
[48] F. Bernardeau, Skewness and Kurtosis in large scale cosmic fields, Astrophys. J. 433 (1994) 1 [astro-ph/9312026].

[49] R. Takahashi, Third Order Density Perturbation and One-loop Power Spectrum in a Dark Energy Dominated Universe, Prog. Theor. Phys. 120 (2008) 549 [0806.1437].

[50] E. Sefusatti and F. Vernizzi, Cosmological structure formation with clustering quintessence, JCAP 03 (2011) 047 [1101.1026].

[51] M. Fasiello and Z. Vlah, Nonlinear fields in generalized cosmologies, Phys. Rev. D 94 (2016) 063516 [1604.04612].

[52] Y. Donath and L. Senatore, Biased Tracers in Redshift Space in the EFTofLSS with exact time dependence, JCAP 10 (2020) 039 [2005.04805].

[53] M. Shoji and E. Komatsu, Massive Neutrinos in Cosmology: Analytic Solutions and Fluid Approximation, Phys. Rev. D 81 (2010) 123516 [1003.0942].

[54] M. Archidiacono, S. Hannestad and J. Lesgourgues, What will it take to measure individual neutrino mass states using cosmology?, JCAP 09 (2020) 021 [2003.03354].

[55] C.-P. Ma and E. Bertschinger, Cosmological perturbation theory in the synchronous and conformal Newtonian gauges, Astrophys. J. 455 (1995) 7 [astro-ph/9506072].

[56] J. Lesgourgues and T. Tram, The Cosmic Linear Anisotropy Solving System (CLASS) IV: efficient implementation of non-cold relics, JCAP 09 (2011) 032 [1104.2935].

[57] M. Archidiacono and S. Hannestad, Efficient calculation of cosmological neutrino clustering in the non-linear regime, JCAP 06 (2016) 018 [1510.02907].

[58] Y. Y. Wong, Higher order corrections to the large scale matter power spectrum in the presence of massive neutrinos, JCAP 10 (2008) 035 [0809.0693].

[59] S. Saito, M. Takada and A. Taruya, Impact of massive neutrinos on nonlinear matter power spectrum, Phys. Rev. Lett. 100 (2008) 191301 [0801.0607].

[60] J. Lesgourgues, S. Matarrese, M. Pietroni and A. Riotto, Non-linear Power Spectrum including Massive Neutrinos: the Time-RG Flow Approach, JCAP 06 (2009) 017 [0901.4550]. 